\title{
The deacylase SIRT5 supports melanoma viability by influencing chromatin dynamics
}

\begin{abstract}
William Giblin, ${ }^{1,2}$ Lauren Bringman-Rodenbarger, ${ }^{1}$ Angela H. Guo, ${ }^{1}$ Surinder Kumar, ${ }^{1}$ Alexander C. Monovich, ${ }^{1}$ Ahmed M. Mostafa, ${ }^{1,3}$ Mary E. Skinner, ${ }^{1}$ Michelle Azar, ${ }^{1}$ Ahmed S.A. Mady, ${ }^{1}$ Carolina H. Chung, ${ }^{4}$ Namrata Kadambi, ${ }^{1}$ Keith-Allen Melong, ${ }^{1}$ Ho-Joon Lee, ${ }^{5}$ Li Zhang, ${ }^{5}$ Peter Sajjakulnukit, ${ }^{5}$ Sophie Trefely, ${ }^{6,7}$ Erika L. Varner, ${ }^{7}$ Sowmya Iyer, ${ }^{8}$ Min Wang, ${ }^{1}$ James S. Wilmott, ${ }^{9}$ H. Peter Soyer, ${ }^{10,11}$ Richard A. Sturm, ${ }^{10}$ Antonia L. Pritchard, ${ }^{12,13}$ Aleodor A. Andea, ${ }^{1,14}$ Richard A. Scolyer, $, 15,16$ Mitchell S. Stark, ${ }^{10}$ David A. Scott, ${ }^{17}$ Douglas R. Fullen, ${ }^{1,14}$ Marcus W. Bosenberg, ${ }^{18}$ Sriram Chandrasekaran,, ${ }^{4,19,20,21}$ Zaneta Nikolovska-Coleska, ${ }^{1,21}$ Monique E. Verhaegen, ${ }^{14}$ Nathaniel W. Snyder, ${ }^{7}$ Miguel N. Rivera, ${ }^{8,22}$ Andrei L. Osterman, ${ }^{17}$ Costas A. Lyssiotis, ${ }^{5,21,23}$ and David B. Lombard ${ }^{1,21,24}$

'Department of Pathology and 'Department of Human Genetics, University of Michigan, Ann Arbor, Michigan, USA. ${ }^{3}$ Department of Biochemistry, Faculty of Pharmacy, Ain Shams University, Cairo, Egypt. ${ }^{4}$ Department of Biomedical Engineering and ${ }^{5}$ Department of Molecular and Integrative Physiology, University of Michigan, Ann Arbor, Michigan, USA. ${ }^{6}$ Department of Cancer Biology, University of Pennsylvania, Perelman School of Medicine, Philadelphia, Pennsylvania, USA. ${ }^{\top}$ Center for Metabolic Disease Research, Department of Microbiology and Immunology, Temple University, Lewis Katz School of Medicine, Philadelphia, Pennsylvania, USA. ${ }^{8}$ Department of Pathology and MGH Cancer Center, Massachusetts Ceneral Hospital and Harvard Medical School, Boston, Massachusetts, USA. ${ }^{9}$ Melanoma Institute Australia, The University of Sydney, Sydney, New South Wales, Australia. ${ }^{10}$ The University of Queensland Diamantina Institute, The University of Queensland, Dermatology Research Centre, Brisbane, Australia. "Department of Dermatology, Princess Alexandra Hospital, Brisbane, Queensland, Australia. ${ }^{12}$ Institute of Health Research and Innovation, University of the Highlands and Islands, An Lóchran, Inverness, United Kingdom. ${ }^{13}$ Oncogenomics, QIMR Berghofer Medical Research Institute, Brisbane, Queensland, Australia. ${ }^{14}$ Department of Dermatology, University of Michigan, Ann Arbor, Michigan, USA. ${ }^{15}$ Tissue Pathology and Diagnostic Oncology, Royal Prince Alfred Hospital, and NSW Pathology, Sydney, New South Wales, Australia. ${ }^{16}$ Faculty of Medicine and Health, The University of Sydney, Sydney, New South Wales, Australia. ${ }^{17}$ Sanford Burnham Prebys Medical Discovery Institute, La Jolla, California, USA. ${ }^{18}$ Departments of Pathology and Dermatology, Yale University School of Medicine, New Haven, Connecticut, USA. ${ }^{19}$ Program in Chemical Biology, ${ }^{20}$ Center for Computational Medicine and Bioinformatics, and ${ }^{21}$ Rogel Cancer Center, University of Michigan Medical School, Ann Arbor, Michigan, USA. ${ }^{22}$ Broad Institute of Harvard and MIT, Cambridge, Massachusetts, USA. ${ }^{23}$ Division of Gastroenterology, Department of Internal Medicine and ${ }^{24}$ Institute of Gerontology, University of Michigan, Ann Arbor, Michigan, USA.
\end{abstract}

Cutaneous melanoma remains the most lethal skin cancer, and ranks third among all malignancies in terms of years of life lost. Despite the advent of immune checkpoint and targeted therapies, only roughly half of patients with advanced melanoma achieve a durable remission. Sirtuin 5 (SIRT5) is a member of the sirtuin family of protein deacylases that regulates metabolism and other biological processes. Germline Sirt5 deficiency is associated with mild phenotypes in mice. Here we showed that SIRT5 was required for proliferation and survival across all cutaneous melanoma genotypes tested, as well as uveal melanoma, a genetically distinct melanoma subtype that arises in the eye and is incurable once metastatic. Likewise, SIRT5 was required for efficient tumor formation by melanoma xenografts and in an autochthonous mouse Braf Pten-driven melanoma model. Via metabolite and transcriptomic analyses, we found that SIRT5 was required to maintain histone acetylation and methylation levels in melanoma cells, thereby promoting proper gene expression. SIRT5-dependent genes notably included MITF, a key lineage-specific survival oncogene in melanoma, and the c-MYC proto-oncogene. SIRT5 may represent a druggable genotype-independent addiction in melanoma.

Authorship note: LBR, AHG, SK, ACM, AMM, and MES contributed equally to this work Conflict of interest: MWB is a consultant for Eli Lilly and Company. RAS has received fees for professional services from QBiotics, Merck Sharp \& Dohme, GlaxoSmithKline, Bristol-Myers Squibb, Dermpedia, Novartis, Myriad, NeraCare, and Amgen. CAL is an inventor on patents pertaining to KRAS-regulated metabolic pathways, redox control pathways in pancreatic cancer, and targeting GOT1 as a therapeutic approach ("Methods for Diagnosing and Treating Oncogenic KRAS-Associated Can cer," US patent no. 2015126580-A1; "Targeting the Glutamine to Pyruvate Pathway for Treatment of Oncogenic KRAS-Associated Cancer," US patent no. 20190136238; international patent no. W02013177426-A2). CAL is also an author on a provisiona patent application concerning the development of technologies that integrate the metabolic flux assay with cellular high content image analysis. DBL owns the equivalent in voting stock or share of ABBV and GILD.

Copyright: (5) 2021, American Society for Clinical Investigation.

Submitted: April 8, 2020; Accepted: April 29, 2021; Published: June 15, 2021

Reference information: J Clin Invest. 2021;131(12):e138926.

https://doi.org/10.1172/JCl138926.

\section{Introduction}

Cutaneous melanoma remains the most lethal skin cancer. In 2021, there will be an estimated 106,110 new melanoma cases and 7180 melanoma-related deaths in the United States (1). Melanoma incidence is rising (2), and melanoma ranks third among all cancers in terms of years of life lost $(3,4)$. Despite the advent of immune checkpoint and targeted therapies, only about half of patients with advanced melanoma achieve long-term remission, even with optimal immune checkpoint therapy (5). Uveal melanoma represents a genetically and clinically distinct subtype of melanoma that arises in the eye, and currently has no effective treatment options once metastatic (6). New therapeutic strategies for advanced melanoma are urgently needed.

Mammalian sirtuins are a family of $7 \mathrm{NAD}^{+}$-dependent lysine deacylases that regulate diverse processes to promote cellular and organismal homeostasis and stress responses. Among these 
proteins, sirtuin 5 (SIRT5) has remained a somewhat enigmatic and poorly characterized sirtuin. SIRT5 is atypical, in that it lacks robust deacetylase activity, and primarily functions to remove succinyl, malonyl, and glutaryl modifications from lysines on its target proteins, in mitochondria, and throughout the cell, thereby regulating multiple metabolic pathways (7-14).

SIRT5-deficient mice are viable, fertile, and mostly healthy $(15,16)$, with the most prominent effects described to date occurring in the myocardium (17). Sirt5-KO mice are more susceptible to ischemia-reperfusion injury and exhibit impaired recovery of cardiac function compared with WT mice (18). Aged Sirt5-KO mice develop cardiac hypertrophy and mildly impaired ejection fraction (19). Whole-body Sirt5-KOs, but not cardiomyocyte-specific KOs, show increased lethality in response to cardiac pressure overload $(20,21)$. Overall, however, the lack of strong phenotypes associated with SIRT5 loss-of-function in normal tissues has hindered progress in understanding the biological significance of SIRT5 and its target posttranslational modifications.

Multiple sirtuins are now linked to neoplasia, as tumor suppressors and/or oncogenes, in a context-specific manner (22). In the context of melanoma, genetic inhibition of SIRT1 in human melanoma cell lines induces senescence and sensitizes drug-resistant cells to vemurafenib, an FDA-approved therapy for the treatment of BRAF-mutant melanoma (23). Conversely, genetic SIRT2 inhibition results in vemurafenib resistance in $B R A F$-mutant melanoma cells by altering MEK/ERK signaling (24). SIRT3 has likewise been reported to play an oncogenic role in melanoma. Reduction of SIRT3 levels in human melanoma lines results in decreased viability, increased senescence, and impaired xenograft formation (25). SIRT6 is upregulated in melanoma cells and tissue samples, and SIRT6 depletion in melanoma cell lines results in reduced colony formation and proliferation (26). Paradoxically, SIRT6 haploinsufficiency induces resistance to targeted therapies in $B R A F$-mutant melanoma cells by regulating IGF/AKT signaling (27).

The functions of SIRT5 in cancer are not well understood, and are a subject of active investigation (7). For example, SIRT5 promotes chemoresistance in non-small cell lung carcinoma cells by enhancing NRF2 activity and expression of its targets involved in cellular antioxidant defense (28). SIRT5 promotes Warburg-type metabolism in lung cancer cells by negatively regulating SUN2, a member of the linker of nucleoskeleton and cytoskeleton complex (29). SIRT5 suppresses levels of reactive oxygen species (ROS) via desuccinylation of multiple targets (superoxide dismutase 1 , glucose-6-phosphate dehydrogenase, and isocitrate dehydrogenase [IDH] 2), thereby promoting growth of lung cancer cell lines in vitro $(30,31)$. SIRT5 also plays an important role in facilitating tumor cell growth by desuccinylating serine hydroxymethyltransferase 2 (SHMT2), which catalyzes the reversible, rate-limiting step in serine catabolism, providing methyl groups for cellular methylation reactions via one-carbon metabolism (1CM) (32). Another study indicated that SIRT5 promotes hepatocellular carcinoma (HCC) proliferation and invasion by targeting the transcription factor E2F1 (33). Similarly, it was recently reported that SIRT5 suppresses apoptosis by deacetylation of cytochrome C, thereby promoting HCC growth (34). SIRT5 also promotes breast cancer tumorigenesis by desuccinylating and stabilizing glutaminase (35), an enzyme that catalyzes conversion of glutamine to glutamate, which supports the meta- bolic demands of tumorigenesis (36). Another recent publication showed that SIRT5 promotes breast cancer growth in part by suppressing ROS, and described selective SIRT5 inhibitors that markedly impaired tumor growth in vivo (37). In contrast, SIRT5 opposes malignant phenotypes associated with expression of mutant IDH, which generates the novel oncometabolite R-2-hydroxyglutarate, thereby perturbing the epigenome (38). IDH mutant glioma cells show increased protein succinylation, exhibit mitochondrial dysfunction, and are resistant to apoptosis. Ectopically expressed SIRT5 in these cells impaired their growth in vitro and in vivo. Another recent report indicates that SIRT5 inactivates STAT3, thus suppressing mitochondrial pyruvate metabolism in lung cancer (39).

Here, we identify a critical requirement for SIRT5 in melanoma cell survival, through chromatin regulation. In all cutaneous and uveal melanoma cell lines tested, from both humans and mice and with varied genetic drivers, SIRT5 depletion resulted in rapid loss of proliferative capacity and cell death. Likewise, SIRT5 loss reduced melanoma formation in xenograft and autochthonous mouse melanoma models. Via transcriptomic analysis, we identified a core set of genes that responds to SIRT5 depletion. Among these, MITF, an essential lineage-specific transcription factor in melanoma, is downregulated, along with expression of its targets (40). SIRT5 loss is also associated with reduced expression of $c-M Y C$, a welldescribed proto-oncogene that is often overexpressed in metastatic melanoma and melanoma cell lines, which plays an important role in therapeutic resistance $(41,42)$. We link the effects of SIRT5 depletion on gene expression to alterations in histone acetylation and methylation induced by metabolic changes occurring in the context of SIRT5 loss-of-function. Taken together, our results identify SIRT5 as a genotype-independent dependency in melanoma cells, likely exerting its effects via chromatin modifications and gene regulation. Given the modest effects of SIRT5 loss-of-function in normal tissues, SIRT5 may represent an attractive therapeutic target in melanoma and potentially other cancer types.

\section{Results}

The chromosomal region encompassing SIRT5 shows frequent copy number gain in human melanoma. In humans, the SIRT5 gene localizes to chromosome $6 \mathrm{p} 23$. The $6 \mathrm{p}$ region exhibits frequent copy number gain in melanoma, an event associated with an adverse prognosis, both in melanoma (43) and other cancers (44). To confirm that gain of the SIRT5 locus specifically occurs in human melanomas, we mined TCGA (The Cancer Genome Atlas) data (45) using cBioportal, and observed that copy number gain or amplification of SIRT5 was present in 55\% of melanoma cases, whereas SIRT5 deletion or mutation was rare (Figure 1A and Supplemental Figure 1, A and B; supplemental material available online with this article, https://doi.org/10.1172/JCI138926DS1; see complete unedited blots in the supplemental material). Increased SIRT5 copy number also correlated with increased SIRT5 mRNA expression in these samples (Supplemental Figure 1C). In contrast, the presence of extra copies of the other 6 sirtuins was much less common in melanoma (Figure 1A and Supplemental Figure 1A). Activating mutations in BRAF and NRAS represent the most common oncogenic drivers in cutaneous melanoma (46). SIRT5 gain or amplification was observed in melanomas with either driver, and in melanomas with the less common driver mutation, NF1 (Figure 
A Gene Cases ( $N=287)$ Genetic Alteration: TCGA
BRAF $74 \%$ ND NRAS $60 \%$ ND
PTEN 66\% ND MITF $40 \%$ ND NF1 $45 \%$ ND
SIRT1 $63 \%$ 1\%
SIRT2 $37 \% \quad 17 \%$
SIRT3 $43 \% \quad 12 \%$
SIRT4 $32 \%$ 9\%
\begin{tabular}{l|l|l|} 
SIRT5 & $63 \%$ & $55 \%$ \\
SIRT6 & $41 \%$ & $15 \%$
\end{tabular}
\begin{tabular}{l|l|l|} 
SIRT6 & $41 \%$ & $15 \%$ \\
SIRT7 & $47 \%$ & $33 \%$ \\
\hline
\end{tabular}
Any $\mathrm{Amp} /$ Amplification Deep Deletion $=$ Missense Mutation (putative drive
Inframe Mutation (putative driver)
Inframe Mutation (unknown signifi

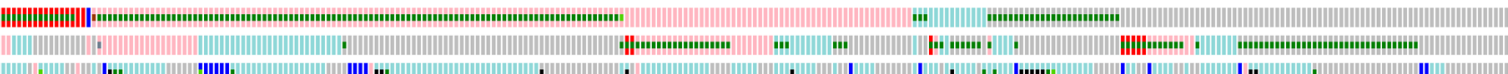

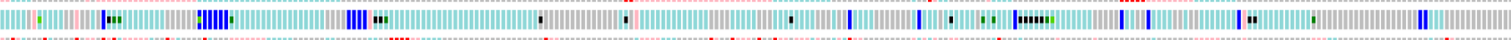

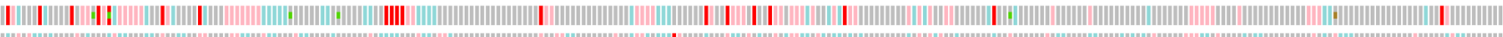

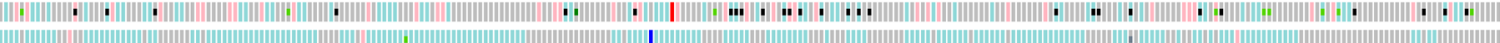 | - . - Gain Gain
-Truncating Mutation (putative driver)
) Truncating Mutation (unknown significance) No alterations in query

B

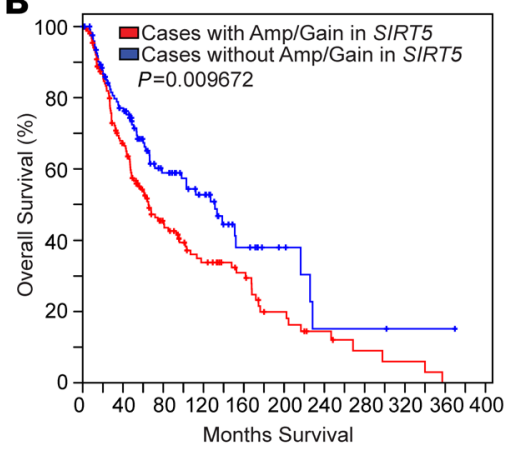

E

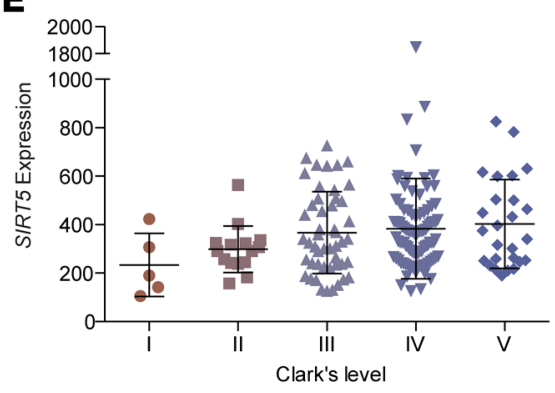

C

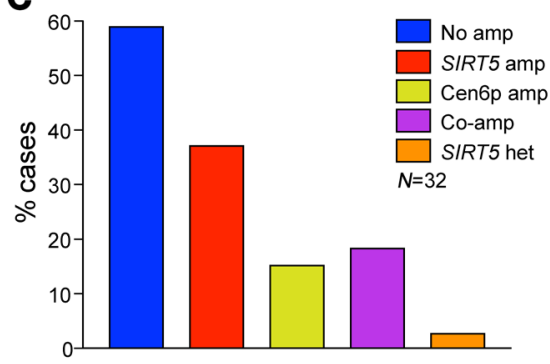

$\mathbf{F}$
D

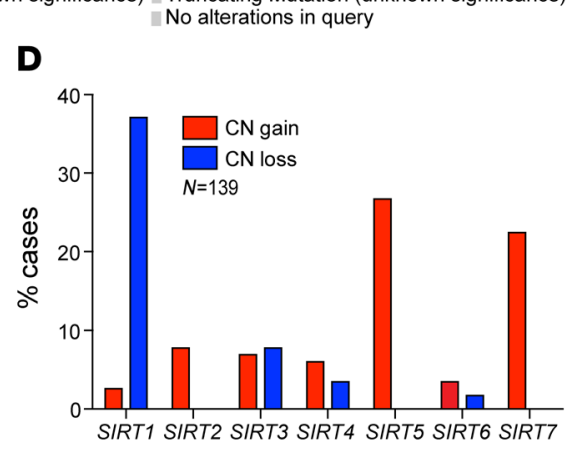

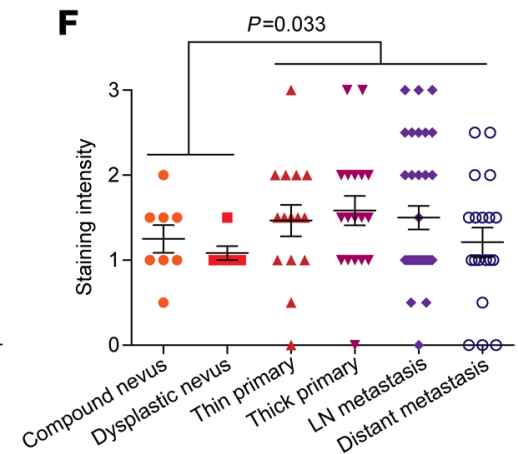

Figure 1. Increased SIRT5 copy number in human melanoma. (A) Gain of extra SIRT5 copies in melanoma. BRAF, NRAS, PTEN, MITF, NF1 and other sirtuins are shown for comparison ( $n=287$; data from TCGA, Provisional, analyzed on cBioPortal). ND, not determined. Percentage of samples with any genomic alteration (Any) or amplification or gain (Amp/Gain) is indicated. Graphed are any alterations queried for the indicated gene. Copy number gain indicates a low-level gain of a single additional copy, and amplification refers to high-level amplification (multiple extra copies). Results from the query (CENE: MUT AMP HOMDEL GAIN HETLOSS) in cBioPortal were analyzed and plotted. (B) Kaplan-Meier analysis of overall survival in melanoma patients with or without copy number gain or amplification of SIRT5. Overall survival was analyzed using the query, "SIRT5: AMP GAIN." (C) SIRT5 (6p23) and centromere 6p (Cen6p) amplification (amp) or coamplification (Co-amp) in melanoma, as assayed by FISH staining ( $n=32$ ). (D) Sirtuin gene copy number (CN) in human melanoma samples, as assayed by high density SNP array $(n=139)$. (E) SIRT5 mRNA expression levels in melanoma correlate with Clark's level $(P=0.0044$, linear regression; $P=0.037,1$-way ANOVA). (F) SIRT5 protein levels are increased in melanoma relative to benign melanocytic lesions $\left(P=0.0333, \chi^{2} ; n=14\right.$ nevi, $n$ = 87 melanoma). See also Supplemental Figure 1 and Supplemental Table 1.

1A). Increased SIRT5 copy number was associated with moderately worsened overall survival ( $P=0.0097$; Figure 1B), although not progression-free survival (Supplemental Figure 1D).

To assess further the status of the SIRT5 locus in melanoma, we performed fluorescence in situ hybridization (FISH) analysis of SIRT5 and the centromere of chromosome 6 in an independent group of melanoma samples (Figure 1C and Supplemental Figure 1E). Consistent with TCGA data, increased SIRT5 copy number was observed in $38 \%(12 / 32)$ of cases analyzed overall, with coamplification of SIRT5 and the centromere of chromosome 6 present in $16 \%$ (5/32) of cases. Similarly, using comparative genomic hybridization analysis in yet another independent group of melanoma samples, gain of the SIRT5 locus was present in $27 \%$ of melanoma cases analyzed (37/139), the most frequent gain among any of the sirtuins (Figure 1D). We also note that the SIRT7 locus was amplified in a substantial fraction of melanoma cases. SIRT7 promotes DNA repair by deacetylating and desuccinylating histones (47); however, it is not currently known what role SIRT7-mediated deacylation might play in melanomagenesis.

We then interrogated SIRT5 mRNA expression in melanomas of varied depth of invasion, and found that increased SIRT5 mRNA expression occurred in melanomas of greater Clark's level, which are more clinically aggressive and confer a worse prognosis (Figure 1E) $(48,49)$. Similarly, we examined SIRT5 protein expression in tissue microarrays containing examples of benign and dysplas- 
A
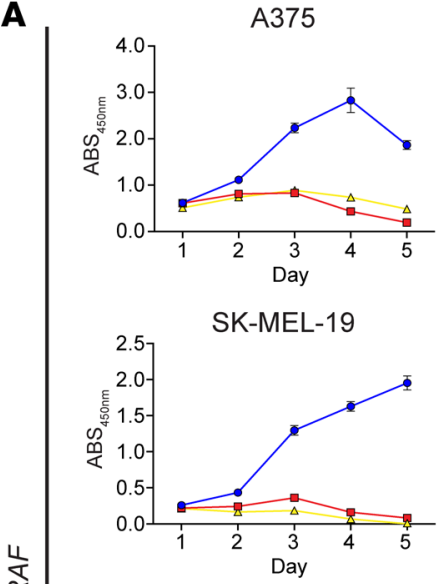

$\frac{4}{\frac{1}{x}}$
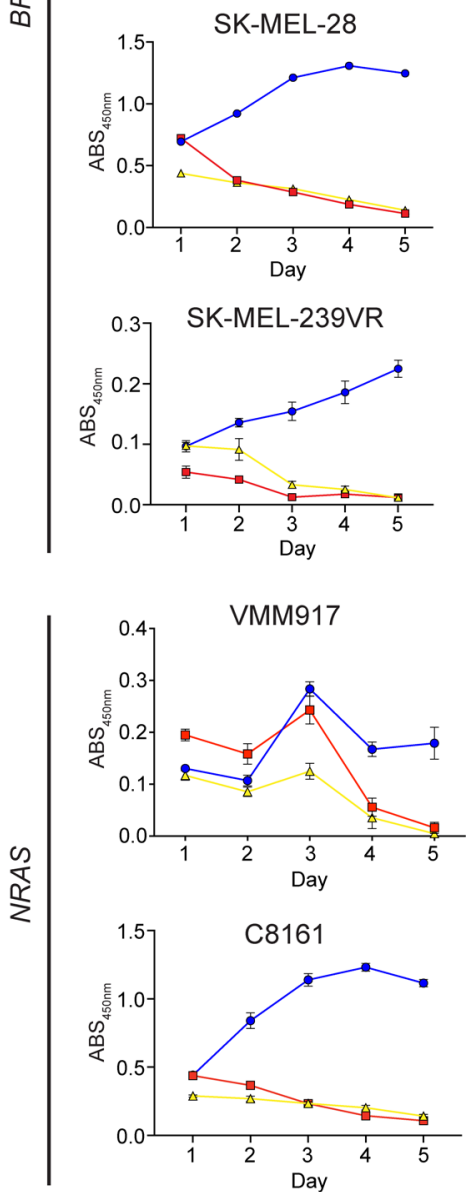

A2058

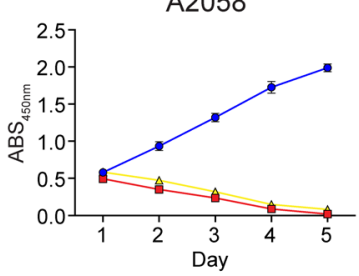

VMM15

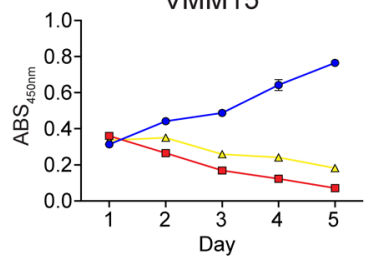

SK-MEL-239
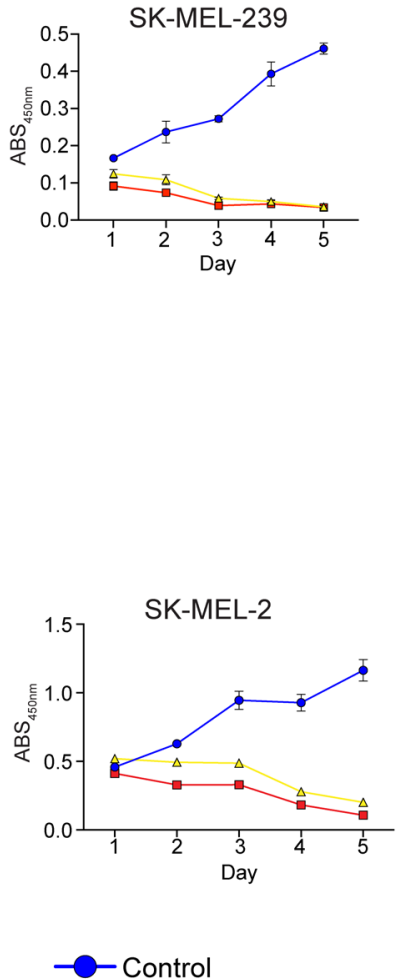

SIRT5 KD1

SIRT5 KD2
B

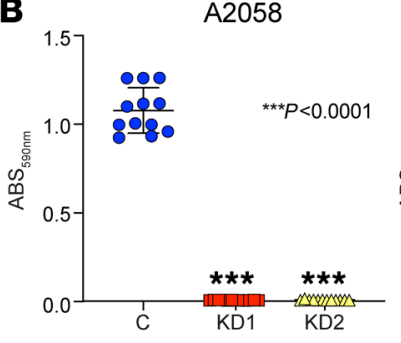

A2058

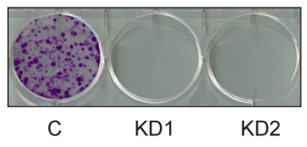

A2058

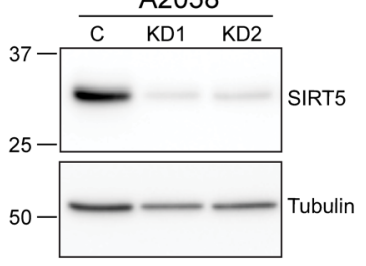

SK-MEL-2

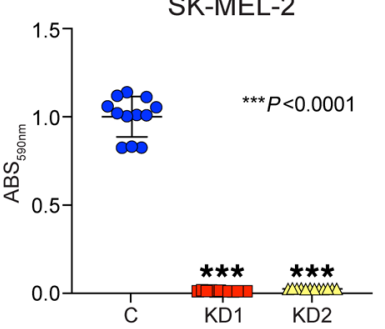

SK-MEL-2
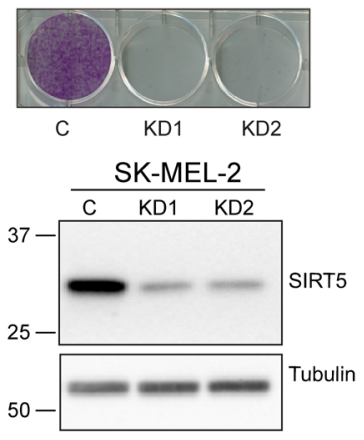

C

A2058
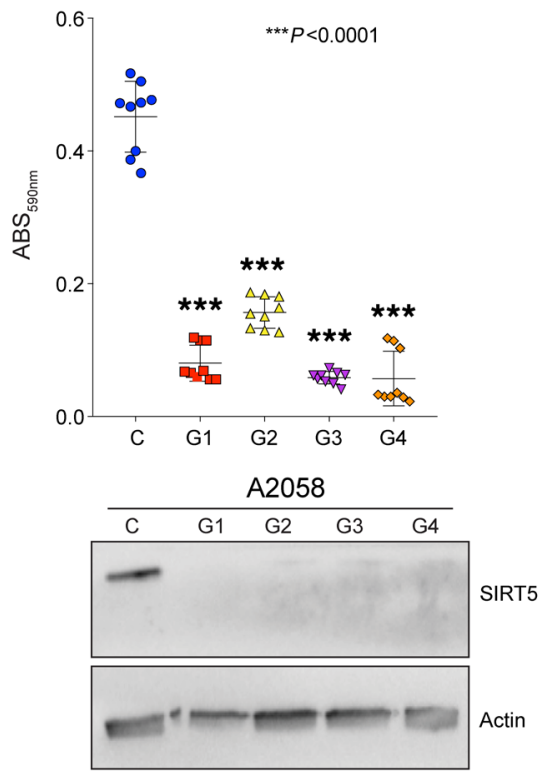

Figure 2. SIRT5 is required for melanoma cell growth and survival. (A) The BRAF or NRAS mutant melanoma cell lines indicated were infected with a nontargeting shRNA (control) or 1 of 2 SIRT5 shRNAs (KD1 or KD2). Equivalent cell numbers were then plated 48 hours after transduction into 96 -well plates in the presence of puromycin. Cell mass was determined at the indicated time points via WST-1 assay, with absorbance measured at 450 nm. Average results ( $n=6$ /time point) are graphed. Error bars represent standard deviation. Representative of 5 of 5 SIRT5 shRNAs tested (see also Figure 3B). (B) SIRT5 KD results in significantly $(P<0.0001,1$-way ANOVA) impaired colony formation by A2058 and SK-MEL-2 cells 12 days after transduction. Cell mass was assayed using crystal violet staining, with absorbance measured at $590 \mathrm{~nm}$. Average of $n=12$ technical replicates is plotted. Error bars represent standard deviation. Representative $(n=4)$ crystal violet-stained wells are shown. Bottom, representative immunoblot analysis demonstrating SIRT5 KD. (C) Top, viability of A2058 cells transfected with the indicated CRISPR guide RNA (Control or G1-C4). Cell mass was assayed using crystal violet staining, with absorbance measured at $590 \mathrm{~nm}$. Average of $n=9$ technical replicates is plotted. Error bars represent standard deviation. Significance calculated using 1-way ANOVA. Bottom, representative immunoblot analysis confirming CRISPR-mediated SIRT5 loss (Control: empty vector).

tic nevi, as well as localized and metastatic melanomas. We found by immunohistochemistry that SIRT5 protein was overexpressed in melanomas relative to benign melanocytic lesions (Figure 1F).
To characterize the stage of melanogenesis at which SIRT5 gain occurs, we screened a panel of genomically characterized benign and dysplastic nevi ( $n=30$; ref. 50) for SIRT5 somatic 
A

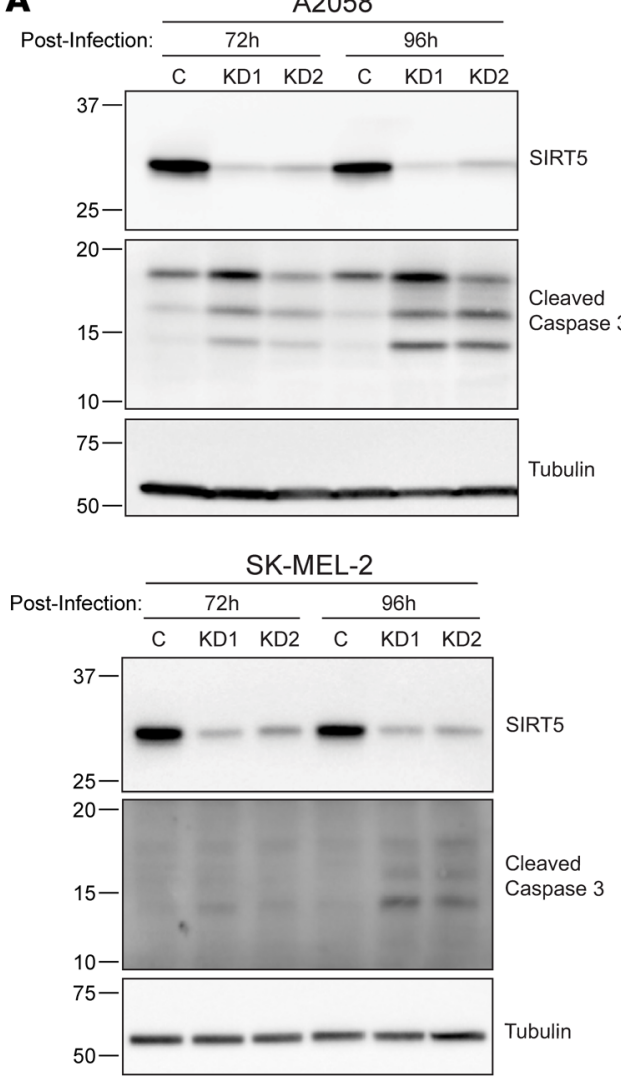

B

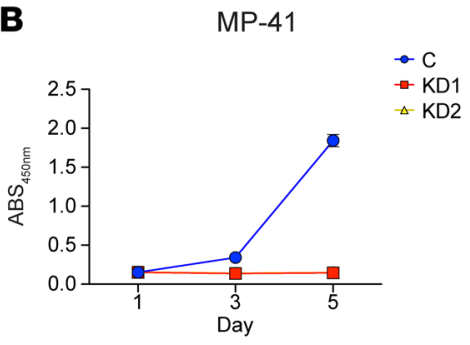

A2058

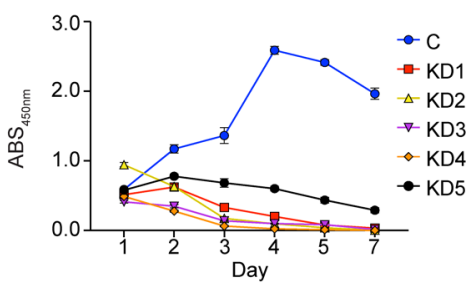

YUMM 5.2

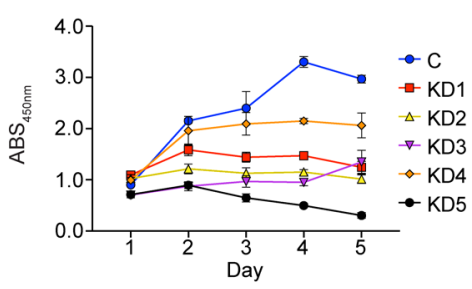

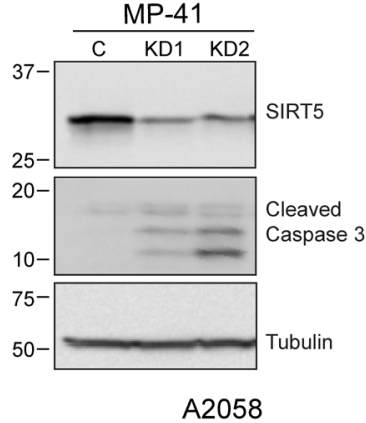

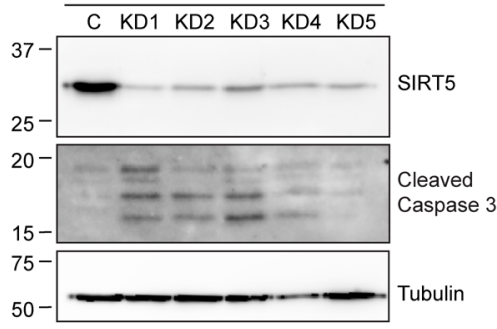

YUMM 5.2

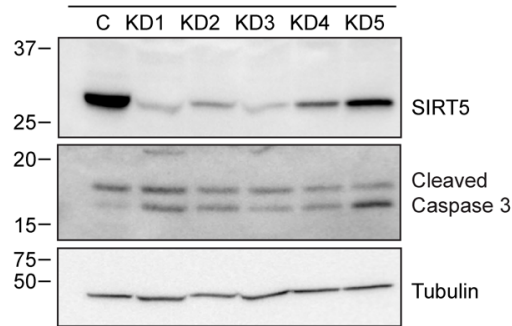

C

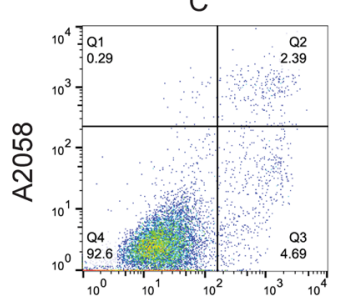

KD1

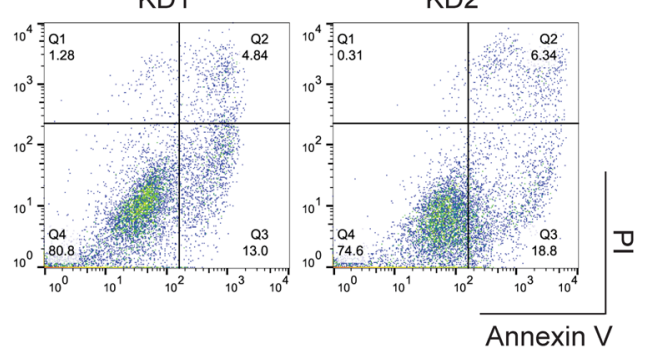

D

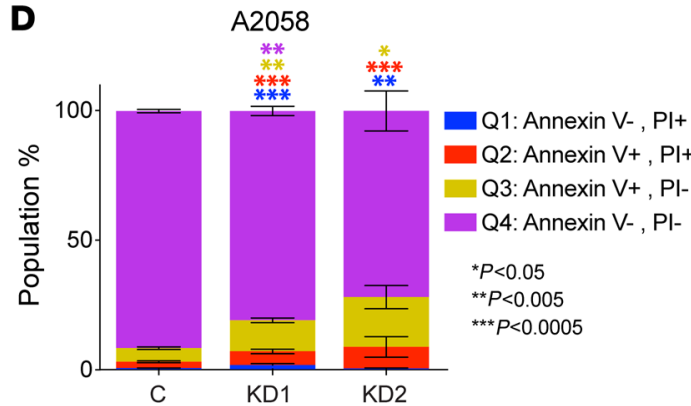

Figure 3. SIRT5 depletion rapidly induces apoptosis in melanoma cells. (A) Immunoblot analysis demonstrating induction of caspase 3 cleavage 72 and 96 hours after transduction with shRNAs SIRT5-KD1 -KD2 in A2058 and SK-MEL-2 cell lines. (B) Viability of MP-41, A2058, or YUMM5.2 cells infected with control (C) or 1 of 5 SIRT5 shRNAs (KD1-KD5) against human SIRT5 (top and middle panels) or mouse Sirt5 (bottom panel). Average results ( $n=6 /$ time point) are graphed. Error bars represent standard deviation. Right panels: immunoblot analysis demonstrating loss of SIRT5 and induction of caspase 3 cleavage following SIRT5 KD. (C) Flow cytometric analysis of A2058 cells stained with Annexin V and propidium iodide (PI), as indicated, showing an increased fraction of Annexin V-positive cells 96 hours after SIRT5 KD. (D) Average of $n=3$ technical replicates is plotted. Error bars represent standard deviation. Significance calculated using unpaired 2-tailed Student's $t$ test. Increased Annexin $\mathrm{V}^{+}$staining is observed in both the PI-positive and PI-negative populations.

mutations and copy number aberrations. No deleterious point mutations were identified in SIRT5; however, there was evidence of regional loss of heterozygosity encompassing the SIRT5 locus in 3 of 30 benign nevi (10\%) assayed (Supplemental Table 1). However, no SIRT5 copy number gain or amplification was identified in any of the nevus samples, supporting the idea that SIRT5 amplification represents a relatively late event in melanomagenesis. This is consistent with the known rarity of such genomic events in nevi $(50,51)$. Overall, these data show that gain or amplification of SIRT5 is a common genomic event in melanoma but not nevi.
SIRT5 is required for survival of BRAF ${ }^{V 600 E}$ and NRAS ${ }^{\mathrm{Q61R}}$ melanoma cells. We assessed the potential requirement of SIRT5 in melanoma cells using a panel of $10 B R A F$ or NRAS mutant melanoma cell lines (Supplemental Table 2). SIRT5 protein was readily detectable by immunoblot in all cell lines tested (Supplemental Figure 2A). We initially depleted SIRT5 using 2 lentiviral shRNAs targeting distinct regions of the SIRT5 mRNA (knockdown 1 [KD1] and KD2; ref. 11). Although predominantly mitochondrial, SIRT5 is also present in the cytosol and the nucleus (11), and was efficiently depleted from all of these compartments upon 
A
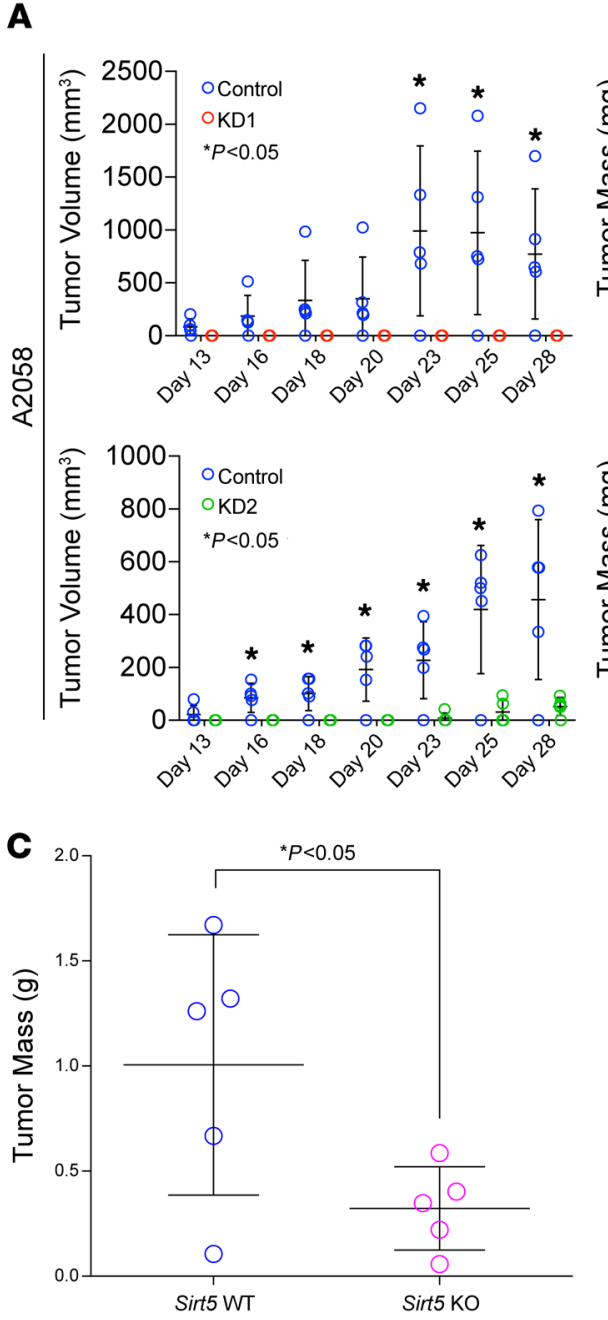
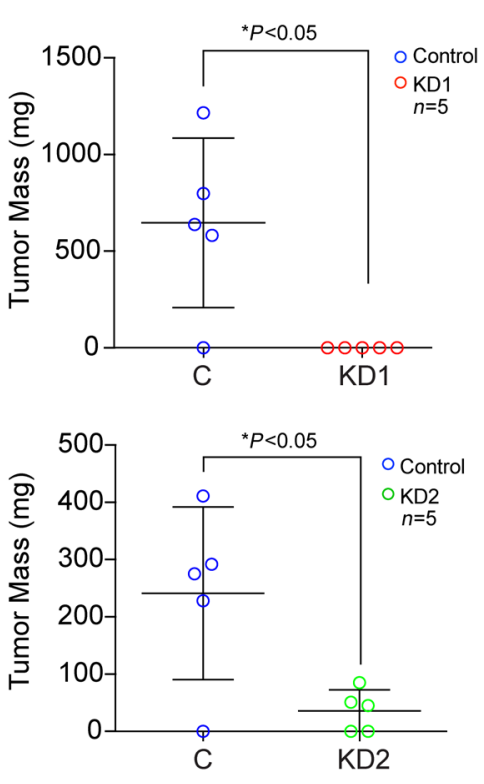

D

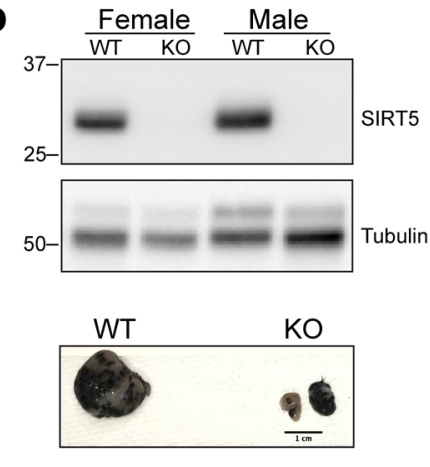

B
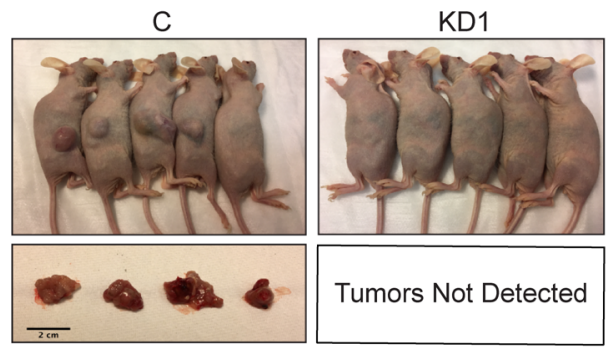

C
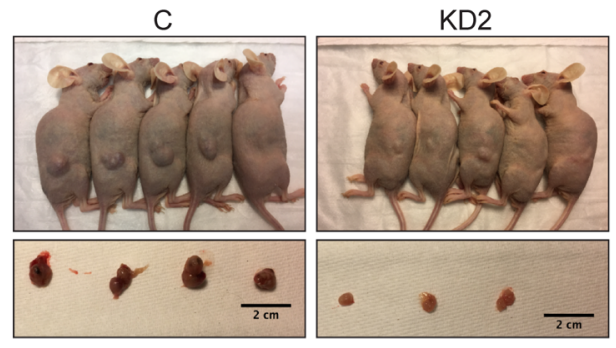

Figure 4. SIRT5 loss-of-function inhibits melanoma tumor growth in vivo. (A) SIRT5 depletion in A2058 cells results in attenuated xenograft tumor growth. Quantification of tumor size was initiated on day 13 after initial injection of cells (left panel). Tumor size was recorded with Vernier calipers on the days indicated. Each point represents the measurements on $n=5$ mice for each condition (C, KD1, or KD2). Pairwise representation of endpoint tumor size in each mouse within each group is plotted (right panel). Average tumor mass measurements at day 28 are plotted $(P<0.05$, paired 2-tailed $t$ test for each group). Error bars represent standard deviation. (B) Mice were sacrificed, and tumors were dissected at 28 days after initial injection. Scale bar below tumors: $2 \mathrm{~cm}$. (C) SIRT5 deficiency attenuates tumor formation in an autochthonous melanoma model. Sirt5-deficient mice were bred into the Braf ${ }^{C A}$ Pten $^{f l / f l}$ Tyr:CreER background (55). Melanomas were induced in littermate male Sirt5-WT or Sirt5-KO mice as shown by topical application of $4 \mathrm{HT}$ at ages 4 to 9 weeks; tumors were weighed following euthanasia. Averages of 5 sets of male mice are plotted $(P<0.05$, paired 2-tailed $t$ test). Mean \pm standard deviation are shown. (D) SIRT5 immunoblot of a representative tumor from a Sirt5-WT or -KO male or female mouse (left). Representative tumor from a Sirt5-WT or KO male mouse, as indicated, after 4HT induction (right). Scale bar: $1 \mathrm{~cm}$.

SIRT5 shRNA transduction in all cell lines tested (Supplemental Figure 2, B and C). In both BRAF ${ }^{\mathrm{V} 600 \mathrm{E}}$ and NRAS ${ }^{\mathrm{Q} 61 \mathrm{R}}$ cells, SIRT5 depletion induced rapid loss of proliferation over the course of 7 days (Figure 2A and Supplemental Figure 2D). Similar results were obtained in an in vitro colony forming assay (Figure $2 \mathrm{~B}$ ). Vemurafenib is a targeted therapy FDA-approved for treatment of BRAF-mutant melanoma. Patients treated with targeted therapies often rapidly relapse with drug-resistant disease (52). SIRT5 inhibition in a vemurafenib-resistant derivative of the melanoma cell line SK-MEL-239, SK-MEL-239VR, induced rapid loss of proliferation upon SIRT5 KD, indicating that these vemurafenib-resistant cells retained SIRT5 dependency (Figure 2A and Supplemental Figure 2E). To complement shRNA-based studies, and to further evaluate the requirement of melanoma cells for SIRT5, we mutated the SIRT5 locus via CRISPR-Cas9, using 4 distinct guide RNAs (gRNAs, G1-G4) targeting SIRT5. Consistent with results obtained using shRNA, a dramatic reduction in colony formation was observed in SIRT5 mutant populations compared with control (Figure 2C). In contrast, SIRT5 KD in several ovarian cancer cell lines did not induce loss of viability as seen in melanoma, indicating that SIRT5 depletion is tolerated in some cancer types (Supplemental Figure 2, F and G).

Loss of SIRT5 leads to apoptotic cell death in cutaneous and uveal melanoma cells. We evaluated the mechanism of cellular attrition induced by SIRT5 loss-of-function. SIRT5 depletion in melanoma cells induced cleavage of caspase 3 (Figure 3, A and B) and induction of Annexin V positivity (Figure 3, C and D). Importantly, SIRT5 depletion also blocked proliferation and induced cleavage 
A

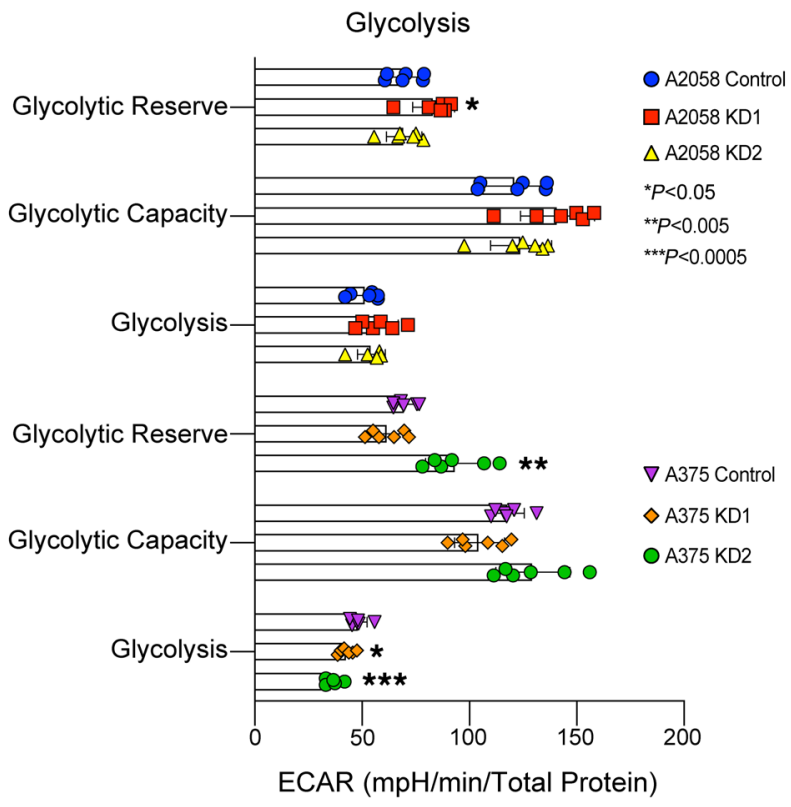

B

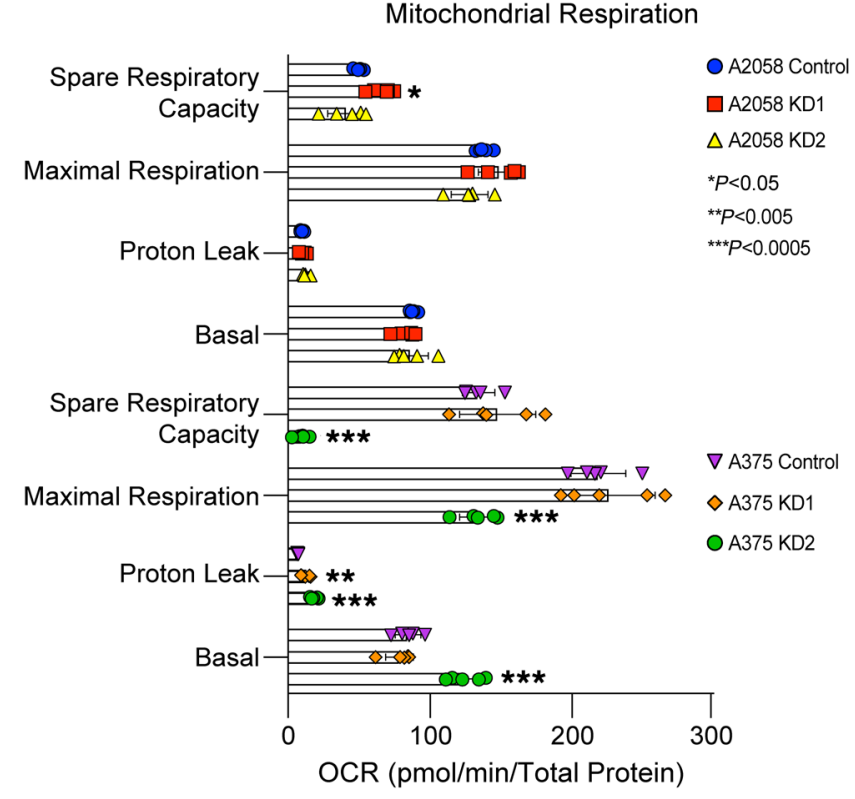

C

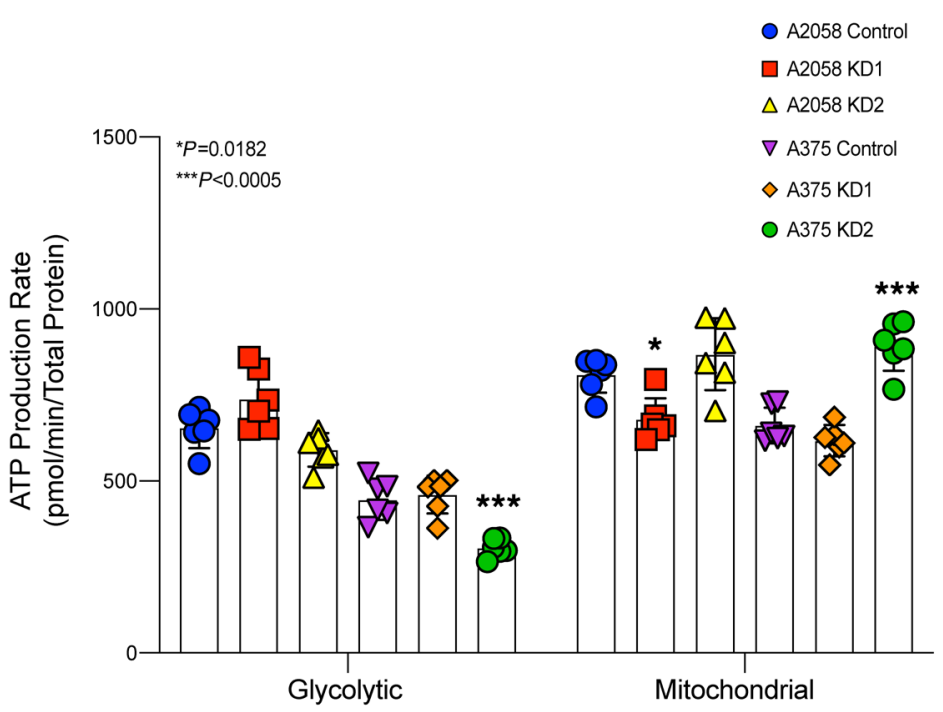

D Mitochondrial

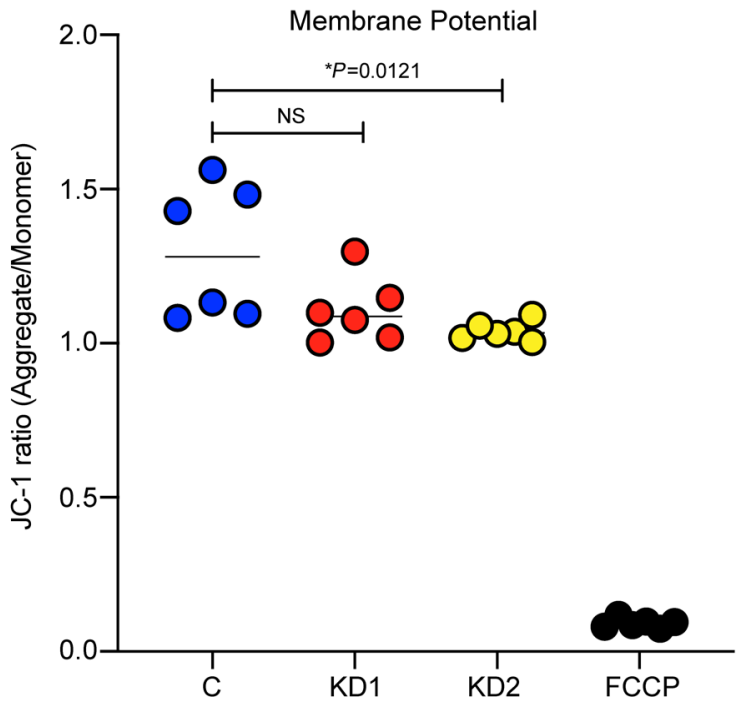

Figure 5. Bioenergetics are maintained upon SIRT5 loss in melanoma cells. A2058 and A375 cells maintain glycolytic function (A), glucose-dependent mitochondrial respiration (B), and ATP production (C) upon SIRT5 depletion compared with control cells. Mitochondrial respiration, glycolytic stress tests, and ATP production rates were measured at 72 hours after transduction with shRNAs against SIRT5 using a Seahorse XFe96 Analyzer. All rates are normalized to total protein content per sample ( $n=6$ for $\mathbf{A}$ and $\mathbf{C}, n=5$ for B). OCR, oxygen consumption rate; ECAR, extracellular acidification rate. (D) Mitochondrial membrane potential is stable in A2058 cells after SIRT5 loss (C, control cells, $n=6$ ). Cells were incubated with JC-1, a dye that exhibits membrane potential-dependent accumulation in mitochondria, indicated by a fluorescence emission shift from green to red. Mitochondrial depolarization is indicated by a decrease in the red/green (aggregate/monomer) fluorescence intensity ratio. FCCP, a mitochondrial uncoupler, depolarizes mitochondrial membrane potential and is used as a positive control. Error bars represent standard deviation. Significance calculated using 1-way ANOVA.

of caspase 3 in uveal melanoma cell lines (Figure 3B; top panel; representative of 4 of 4 uveal melanoma cell lines tested; see Supplemental Table 2). Cell loss and induction of caspase 3 cleavage at 96 hours after transduction were also observed, to varying degrees, with an additional 3 unique shRNAs targeting human SIRT5 (Figure 3B; middle panel), and 5 unique shRNAs targeting murine Sirt5 in YUMM5.2, a mouse melanoma cell line (ref. 53 and Figure 3B; bottom panel).
Nonapoptotic mechanisms of cell death have been described in melanomas and other cancer types, specifically: autophagic, ER-stress induced, necroptosis and pyroptosis (54). We evaluated whether SIRT5 KD in melanoma cell lines harboring either BRAF or NRAS mutations induced these alternate cell death pathways. We did not observe increased conversion of LC3 A/B I to LC3 A/B II or SQSTM1/p62 loss (Supplemental Figure 3A), increased expression of PERK, Cal- 
A

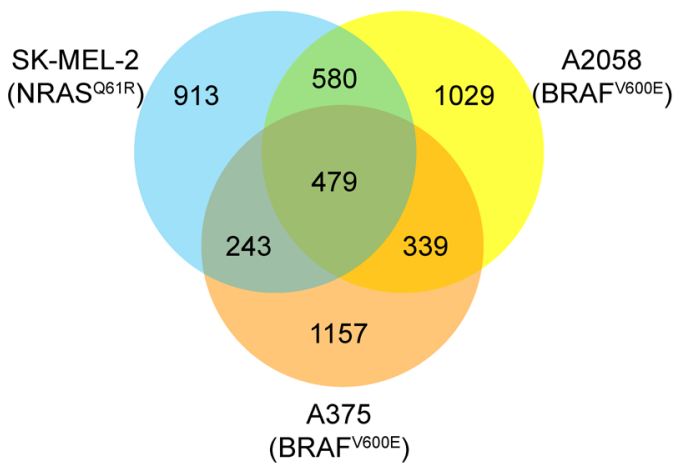

B Downregulated

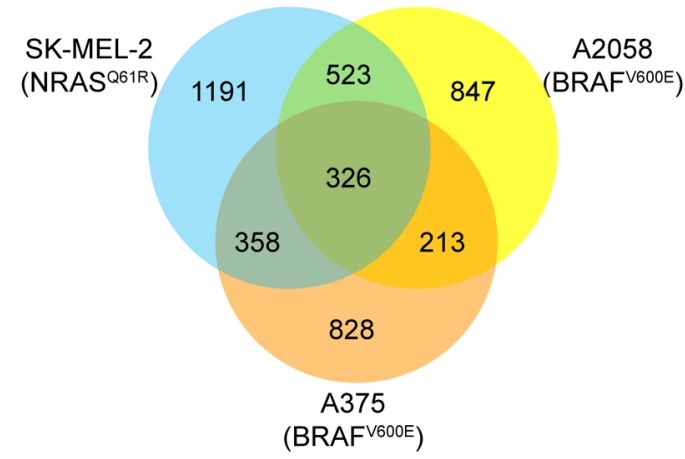

C

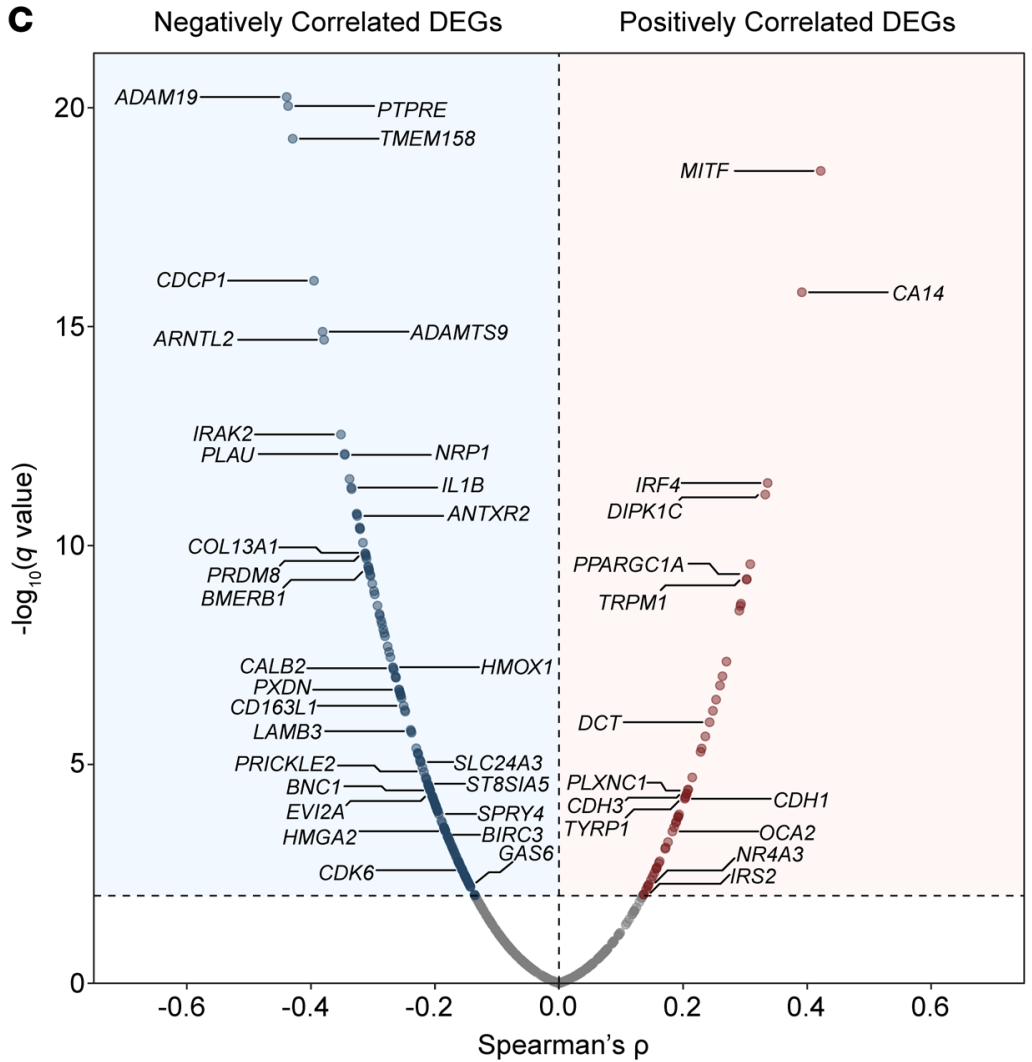

D
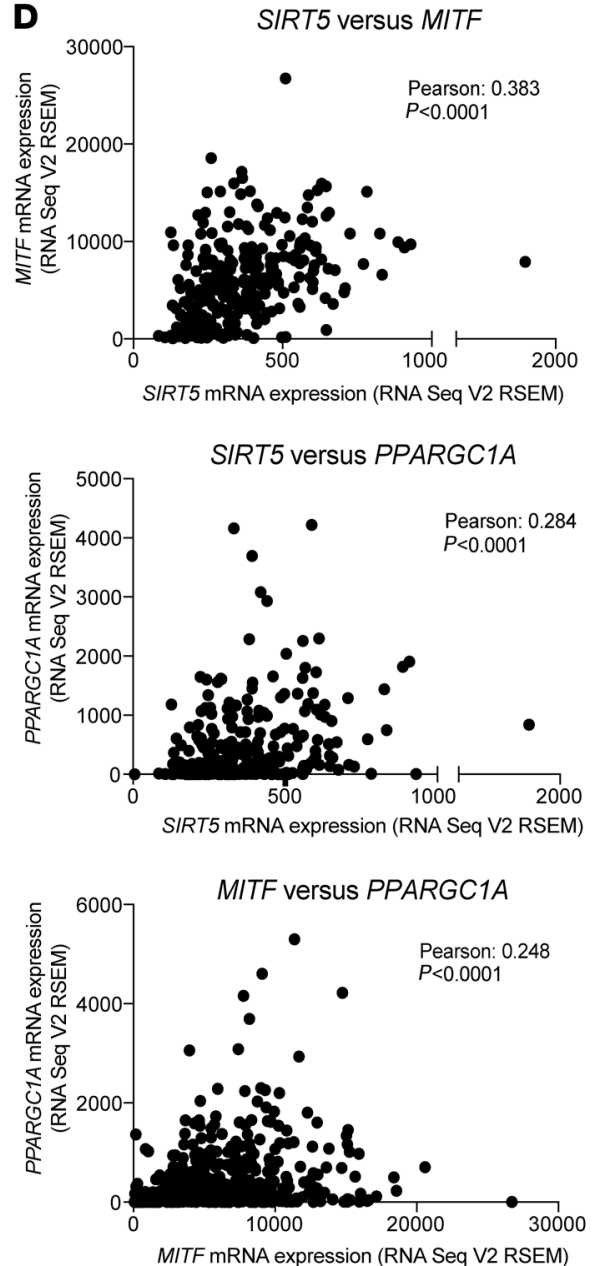

Figure 6. Transcriptomic analysis reveals MITF dependency on SIRT5 expression. Genes (A) upregulated or (B) downregulated upon SIRT5 KD. Only genes significantly $(P<0.05)$ altered in both KDs in each cell line, as indicated, were scored. (C) Expression levels of DEGs (qadj $<0.05)$ in response to SIRT5 KD were correlated with SIRT5 gene expression using Spearman's rank correlation coefficient in 443 sequenced human skin cutaneous melanoma (SKCM) samples, identifying DEGs with significant clinical correlation with SIRT5 expression $(q<0.01)$. Labeled genes represent oncogenes or extremely correlated genes most significantly altered by SIRT5 KD ( $q<0.0001$, $\log _{2}$ fold change $>2$ ). (D) Expression of SIRT5, MITF, and the MITF target, PPARCC1A, are positively correlated in melanoma clinical samples $(P<0.0001$, data from TCCA, analyzed on cBioPortal; see Figure $1 A)$.

nexin, IRE1 alpha or PDI (Supplemental Figure 3B), phosphorylation of either MLKL or RIP (Supplemental Figure 3C), or accumulation of gasdermin D or caspase 1 cleavage products (Supplemental Figure 3D), although there was some variation observed between different SIRT5 KD constructs. Thus, SIRT5 is required for survival and proliferation of multiple genetically diverse melanoma cell lines in vitro, in both human and mouse, and for survival of human uveal melanoma cells. 
A
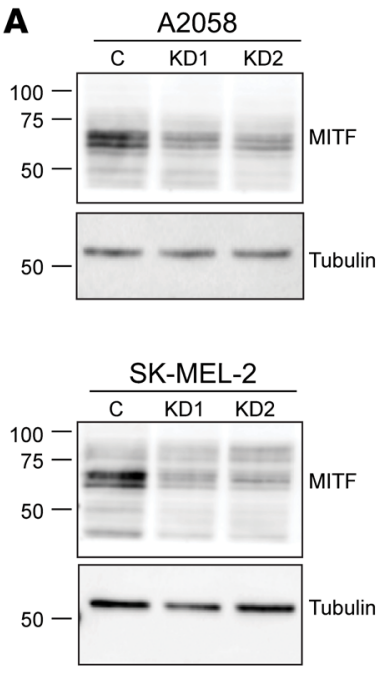

SK-MEL-239

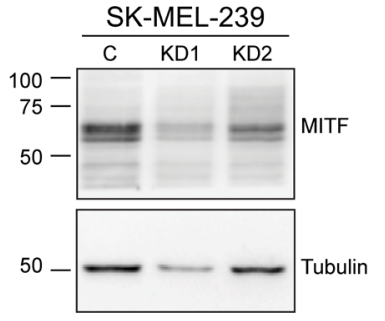

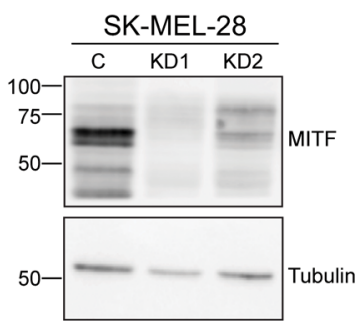
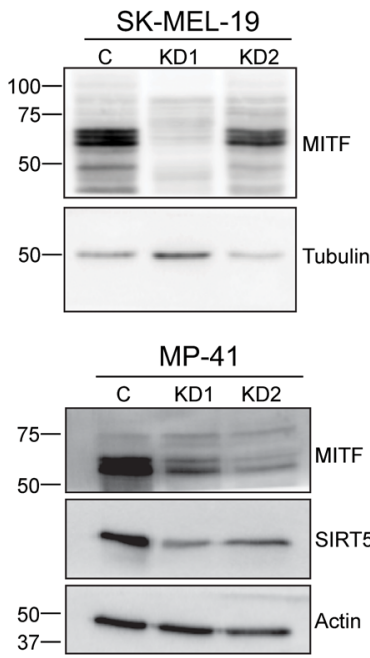

B
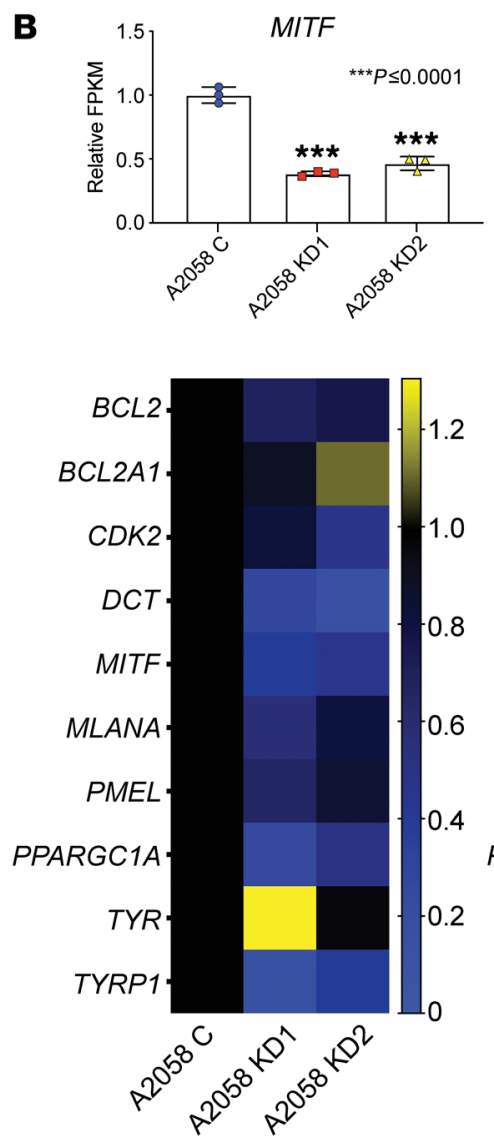
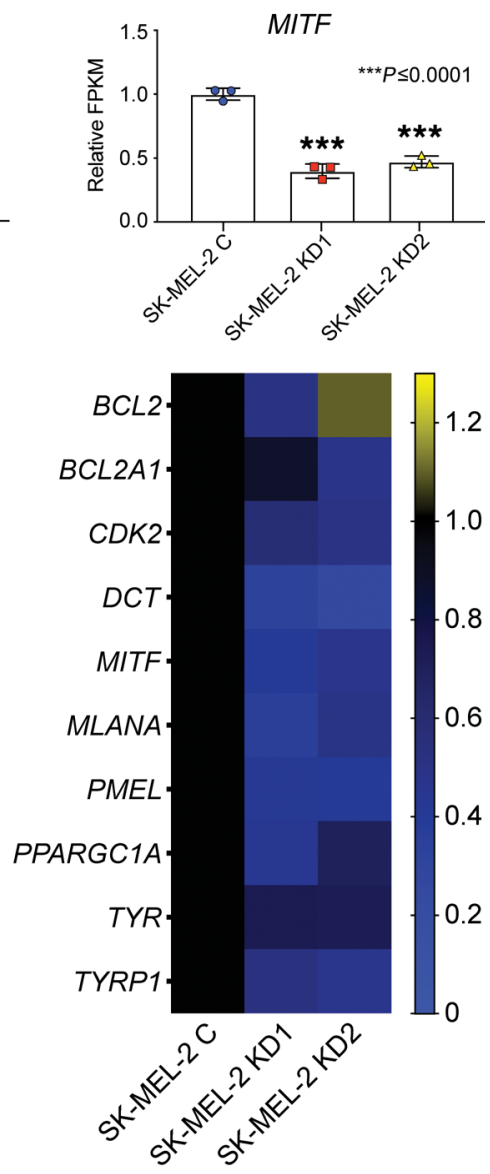

Figure 7. Expression of MITF and MITF target genes is dependent upon SIRT5. (A) Immunoblot demonstrating loss of MITF expression 96 hours after transduction with shRNAs SIRT5-KD1 or -KD2 compared with a nontargeting control in 5 cutaneous and 1 uveal melanoma cell line, as indicated. (B) Relative FPKMs in A2058 and SK-MEL-2 cells demonstrate a loss of MITF (bar graphs, upper panels) and several MITF target gene transcripts upon SIRT5 KD (heatmaps, lower panels). Scale bars adjacent to heat maps indicate linear fold change (control set to 1). Error bars represent standard deviation. Significance calculated using 1-way ANOVA. C, control.

SIRT5 supports robust melanoma tumor formation in vivo. To investigate the potential requirement for SIRT5 to support melanoma tumor development in vivo, we initially employed a xenograft assay. Immediately following transduction with SIRT5 shRNAs, A2058 melanoma cells were subcutaneously injected into the flanks of female NOD/SCID mice (Supplemental Figure 4A). Tumor growth was followed by serial measurement of tumor volume (Figure $4 \mathrm{~A}$; left panel). SIRT5 depletion greatly impaired tumor growth and reduced tumor size at endpoint relative to controls (Figure 4A, right panel, Figure 4B, and Supplemental Figure 4B).

To examine the role of SIRT5 in melanoma development in a more physiologic, immunocompetent context, we crossed Sirt5-KO mice to a commonly used mouse melanoma model, the Braf ${ }^{C A}$ Pten ${ }^{f / f l}$ Tyr:CreER strain (55). Topical application of 4-hydroxytamoxifen (4HT) in this system induces activated BRAF expression and ablation of Pten in melanocytes, resulting in melanoma development. In males, SIRT5-deficient mice showed an approximately 3-fold reduction in tumor mass on average (WT: $1.005 \pm 0.618$ g vs. KO: $0.323 \pm 0.198 \mathrm{~g} ; P<0.05$; Figure $4 \mathrm{C}$ and $4 \mathrm{D})$. In our colony, female mice showed rapid ulceration of even small melanoma tumors following induction (unpublished observation), requiring euthanasia of the host and rendering it difficult to assess the effects of SIRT5 in melanoma in females. Thus, SIRT5 promotes human and mouse melanoma growth, both in cell culture and in vivo.

Neither glucose nor glutamine metabolism are greatly altered by SIRT5 loss. Initially, we considered the possibility that SIRT5 depletion might induce global metabolic collapse and energetic catastrophe in melanoma. SIRT5 has been reported to promote mitochondrial respiration $(56,57)$ and glycolysis $(14)$. We previously showed that SIRT5 suppresses mitochondrial respiration through pyruvate dehydrogenase and complex II in 293T cells and liver mitochondria (11), a finding recapitulated in some systems $(39)$ but not others $(56,57)$. We used the XFe96 Extracellular Flux Analyzer to assess the effects of SIRT5 depletion on cellular bioenergetics in melanoma cells. Relative to SIRT5-proficient controls, SIRT5-KD A2058 or A375 cells did not show consistent changes in the extracellular acidification rate (ECAR), a measure of cellular glycolysis (Figure 5A). Likewise, glucose-dependent mitochondrial oxygen consumption rate (OCR), ATP production, and mitochondrial membrane potential were not consistently affected by SIRT5 depletion (Figure 5, B-D).

Melanoma and many other cancer types replenish the TCA cycle in part via glutaminolysis (58-61). In this pathway, glutaminase (GLS) catalyzes conversion of glutamine to glutamate, gen- 
A

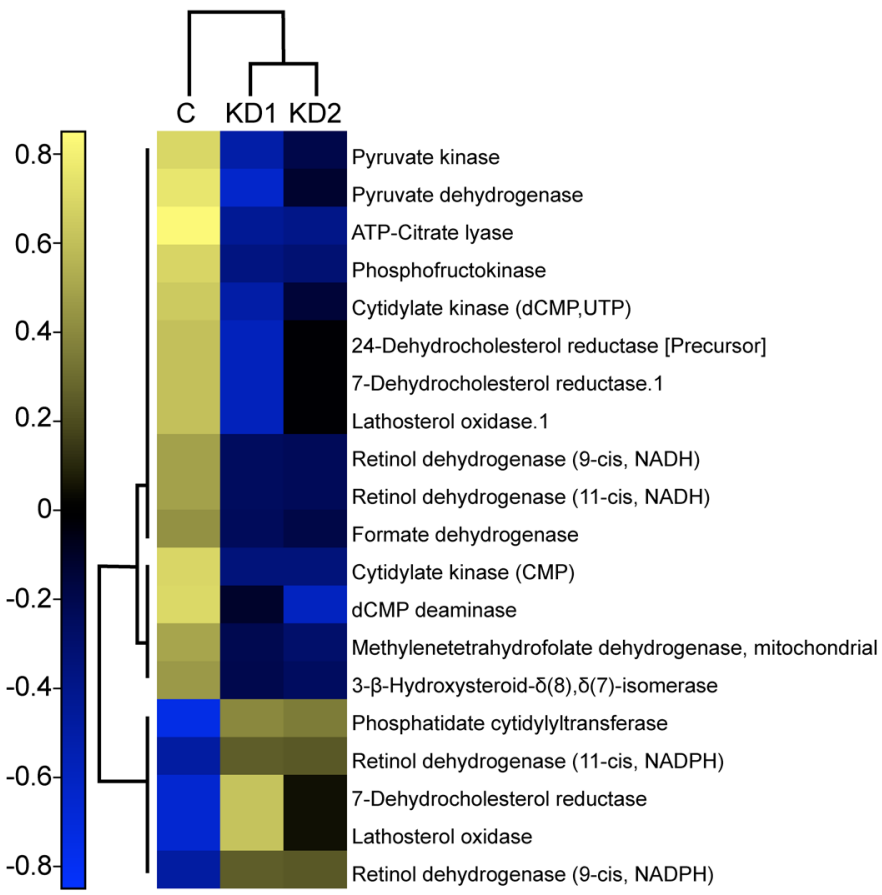

B

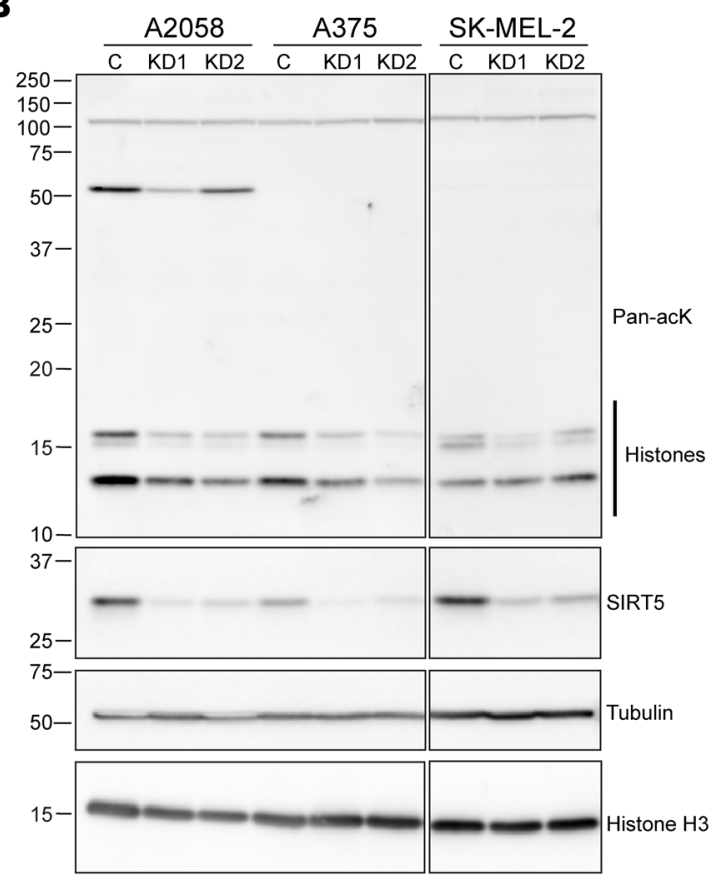

D

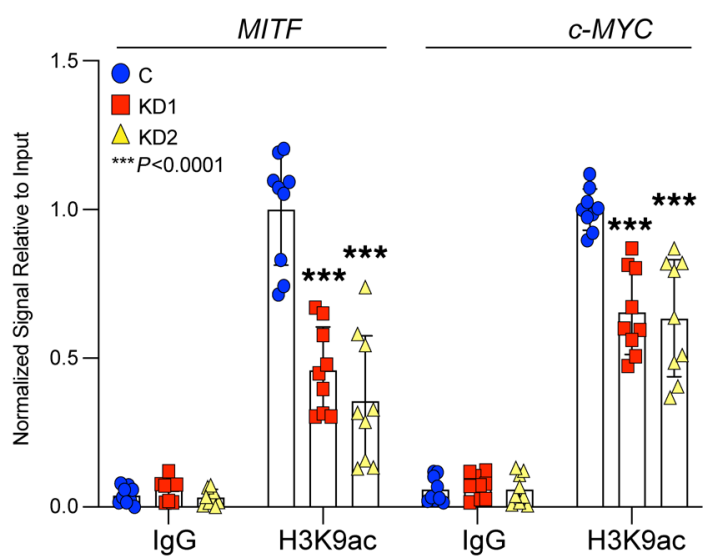

E
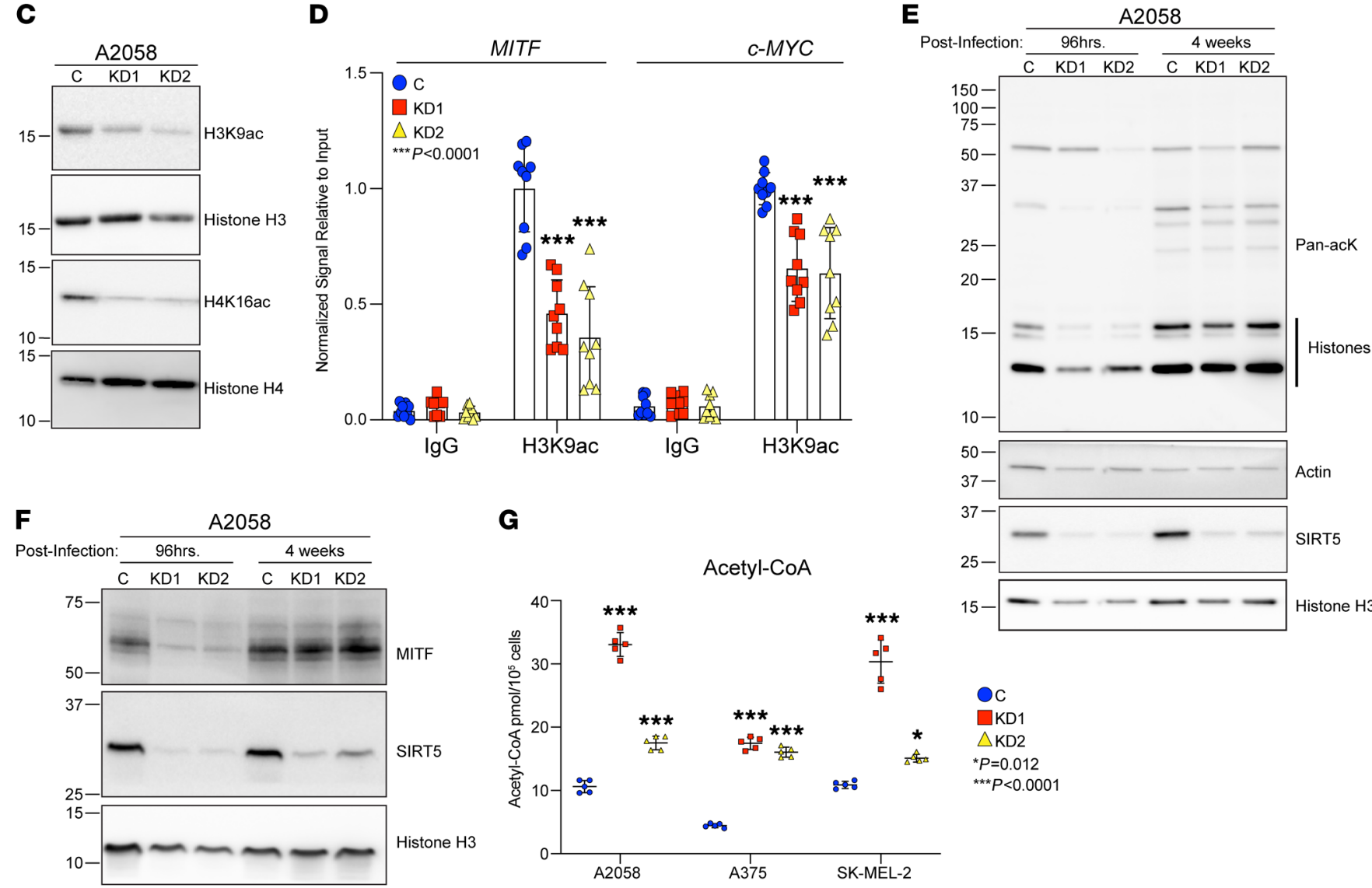

G

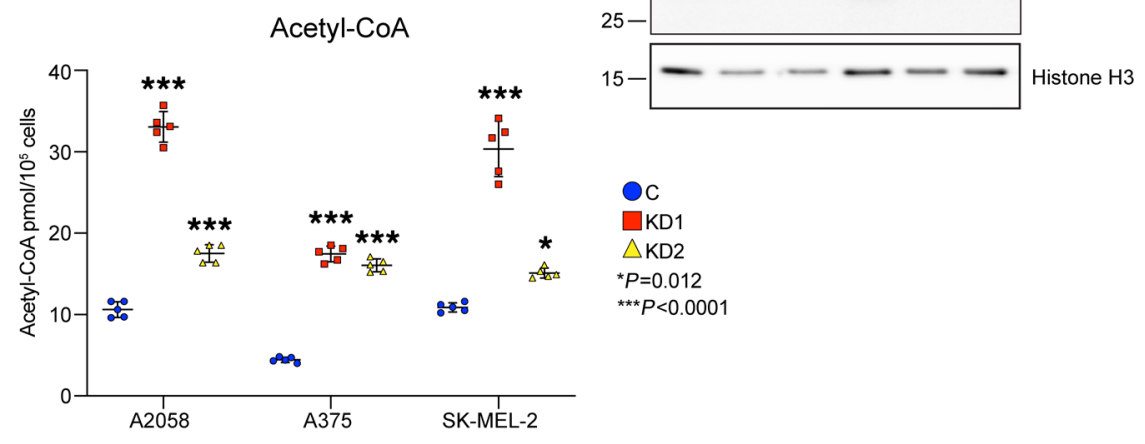


Figure 8. SIRT5 promotes histone acetylation in melanoma. (A) Heatmap of $z$ scores calculated from metabolic reaction fluxes predicted by genomescale modeling to be differentially active $(P<0.01)$ after SIRT5 KD. (B) Total histone acetylation is reduced 96 hours after transduction with shRNAs SIRT5-KD1 or -KD2 compared with a nontargeting control in melanoma cell lines. Lanes were run on the same gel but are noncontiguous. (C) Immunoblot demonstrating loss of $\mathrm{H} 3 \mathrm{~K} 9 \mathrm{ac}$ and $\mathrm{H} 4 \mathrm{~K} 16 \mathrm{ac} 96$ hours after transduction with shRNAs SIRT5-KD1 or -KD2 compared with a nontargeting control in A2058 cells. (D) H3K9ac is reduced within the promoter regions of MITF and c-Myc in SIRT5-depleted A2058 cells via CUT\&RUN followed by qRTPCR. Signal (Ct values) relative to input DNA were normalized to control samples for each primer set. Graphed are averages of $n=9$ replicates. Error bars represent standard deviation. Significance calculated using 1-way ANOVA. Acetylation (E) and MITF expression (F) are restored in A2058 cells lacking SIRT5 after 4 weeks of continual culture in puromycin. (C) Total cellular acetyl-CoA levels are increased in A2058, A375 and SK-MEL-2 cells 96 hours after SIRT5 depletion. Acetyl-CoA abundance was quantified by liquid chromatography-high resolution mass spectrometry and normalized to cell number. Plotted are average $(n=5)$ acetyl-CoA levels as pmol acetyl-CoA $/ 10^{5}$ cells. Error bars represent standard deviation. Significance calculated using 1-way ANOVA. C, control.

erating carbon and nitrogen to fuel the metabolic demands of tumorigenesis. In breast cancer cells, SIRT5 desuccinylates GLS to stabilize it, protecting it from ubiquitination and subsequent degradation. Loss of SIRT5 resulted in decreased GLS expression, exogenous glutamine consumption, glutamine-derived intracellular metabolite levels, and cellular proliferation (35). These findings, along with reports that inhibiting glycolysis or glutamine metabolism sensitizes melanoma cells to cell death, prompted us to investigate a potential role for SIRT5 in promoting glutamine metabolism in melanoma $(62,63)$. We cultured control or SIRT5-KD A2058 cells in medium containing glutamine labeled with stable isotopes $\left(\left[{ }^{15} \mathrm{~N}_{2}\right]\right.$-glutamine or $\left[{ }^{13} \mathrm{C}_{5}\right]$-glutamine) or in medium containing $\left[{ }^{13} \mathrm{C}_{6}\right]$-glucose, and measured both the labeling derived from ${ }^{13} \mathrm{C}$ or ${ }^{15} \mathrm{~N}$ and the total quantities of cellular metabolites. Following SIRT5 KD, the fractional labeling of glutamine-derived metabolites (glutamate, aspartate, and TCA cycle metabolites) modestly decreased when cells were cultured in $\left[{ }^{13} \mathrm{C}_{5}\right]$-glutamine, but showed corresponding (or compensatory) increases in labeling when cultured in ${ }^{13} \mathrm{C}_{6}$-glucose (Supplemental Figure 5, A and B). Labeling derived from ${ }^{15} \mathrm{~N}_{2}$-glutamine was inconsistent between the $2 \mathrm{KD}$ constructs analyzed in A2058 cells (Supplemental Figure 5C). Overall, these results are consistent with previous results showing that SIRT5 promotes glutaminase activity (35). However, importantly, total cellular pools of glutamate, aspartate, TCA cycle, or other metabolites were not consistently reduced by SIRT5 loss (Supplemental Figure 5D), indicating that although the depletion of SIRT5 may reduce glutaminase activity, this effect is insufficient to compromise levels of essential cellular metabolites. In parallel studies, glutamine-dependent mitochondrial OCR and GLS protein levels were assessed, and were not appreciably altered by SIRT5 depletion across multiple melanoma cell lines (Supplemental Figure 5, E and F). Moreover, incubation of SIRT5-KD A2058 cells with exogenous nonessential amino acids plus alpha-ketoglutarate - interventions that can rescue defects in glutamine catabolism (61) - in the context of SIRT5 KD failed to rescue the proliferative defect observed upon SIRT5 loss (Supplemental Figure 5G). Taken together, these data indicate that neither glycolysis nor glutamine metabolism represent major SIRT5 target pathways in promoting melanoma viability.

Transcriptomic analysis reveals a requirement for SIRT5 in supporting MITF and MITF target gene expression. To understand the requirement of melanoma cells for SIRT5 mechanistically, RNASeq-based transcriptomic analysis was performed on 3 cutaneous melanoma cell lines (A2058, A375, and SK-MEL-2), each subjected to SIRT5 depletion using 2 distinct shRNAs (Supplemental Table 3). A gene was scored as differentially expressed only if it was consistently altered in all biological replicates by both independent SIRT5 shRNAs. We identified core sets of protein-coding genes whose expression responded to SIRT5 KD, many of which overlapped among the cell lines (Figure 6, A and B). We then asked if any significant differentially expressed genes (DEGs) found in our SIRT5 RNA-Seq data set correlated with SIRT5 expression in TCGA data of clinical human skin cutaneous melanoma samples (see Methods for details). The most significant positively correlated overlapping DEG in this analysis was the melanocyte inducing transcription factor (MITF) (Figure 6C).

MITF is a key lineage-specific oncogenic transcription factor in melanoma that plays crucial roles in the development and proliferation of melanocytes (40). MITF is expressed in human melanomas, and MITF amplification, present in a subset of melanoma tumors, portends a poor prognosis (64). Melanomas exhibit a wide range of MITF expression levels (65-67). In cutaneous melanoma cells with robust baseline MITF expression, MITF protein and mRNA expression declined markedly in response to SIRT5 $\mathrm{KD}$ (Figure 7, A and B) and associated with decreased expression of MITF's canonical targets: genes involved in metabolism (PPARGC1A), melanocytic differentiation (TYR, MLANA), cell survival (BCL2), and others (Figure 7B). A trend toward a reduced $M I T F$ gene expression profile was also observed in A375 cells, which have low baseline MITF expression, upon SIRT5 KD (Supplemental Figure 6A). Decreased SIRT5, MITF, and MITF target gene expression was validated by qRT-PCR in A2058 cells, and to a lesser degree, in SK-MEL-2 (Supplemental Figure 6B). We also observed a decrease in MITF protein levels upon SIRT5 KD in MP-41 cells, a uveal melanoma line (Figure 7A).

To assess the potential relationship between SIRT5 and MITF in a more physiologic, non-loss-of-function setting, we mined TCGA data to test whether any correlation exists between SIRT5 and MITF mRNA expression in melanoma clinical samples. Consistent with the RNA-Seq data, mRNA coexpression analysis revealed a strong positive correlation between SIRT5, MITF, and 2 canonical MITF target genes, PPARGC1A and BCL2. Indeed, the correlation between SIRT5 and MITF expression was stronger than that of MITF with these 2 of MITF's targets (Figure 6D and Supplemental Figure 6C). As a specificity control, SIRT3 levels showed a modest, negative correlation with MITF expression (Supplemental Figure 6C). These data suggest that SIRT5 expression levels influence expression of MITF and its targets in patient melanoma tumors.

Previous reports demonstrate that the proto-oncogene $c-M Y C$ is upregulated in melanoma tumors and cell lines, acting to bypass mutant BRAF- or NRAS-induced senescence during melanomagenesis (41). Furthermore, siRNA KD of c-MYC in melanocytic tumor cells results in a loss of MITF expression (68). Consistent 
A

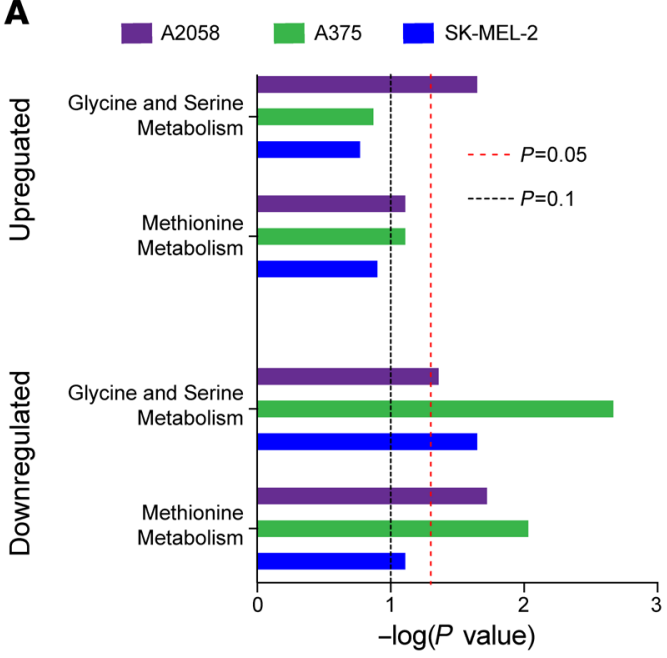

C

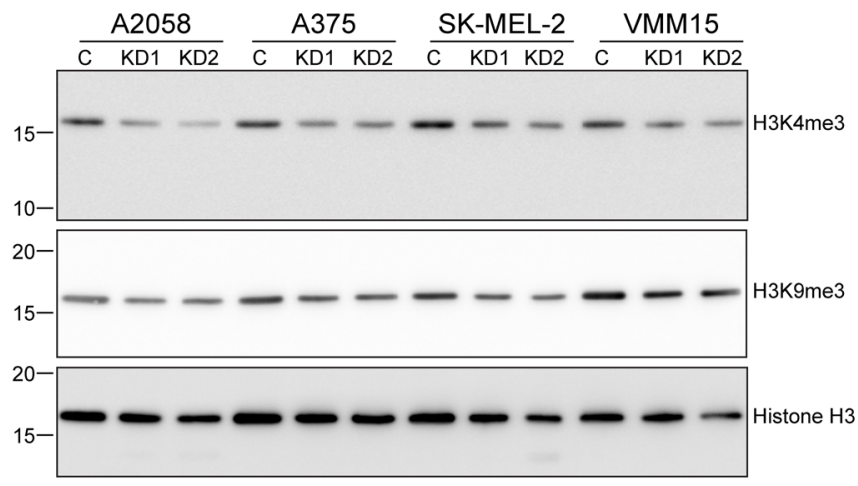

B

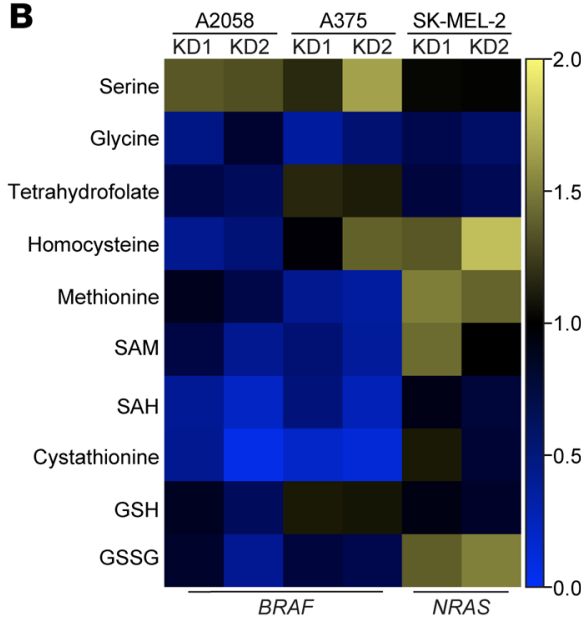

D

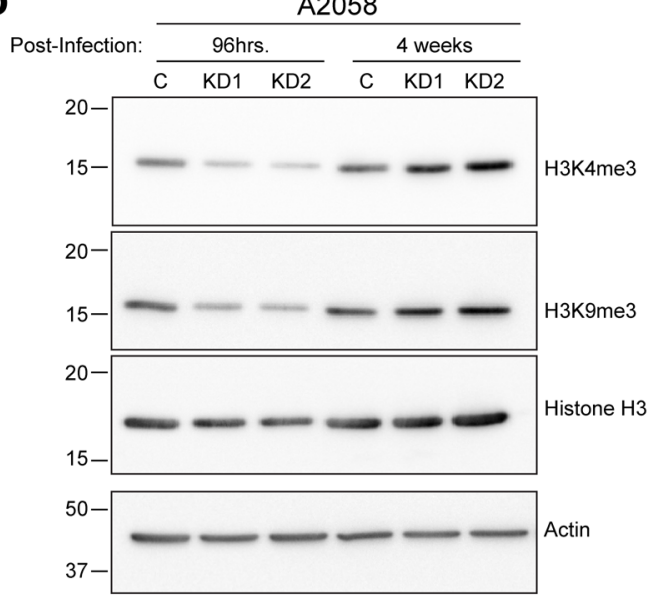

$\mathbf{E}$

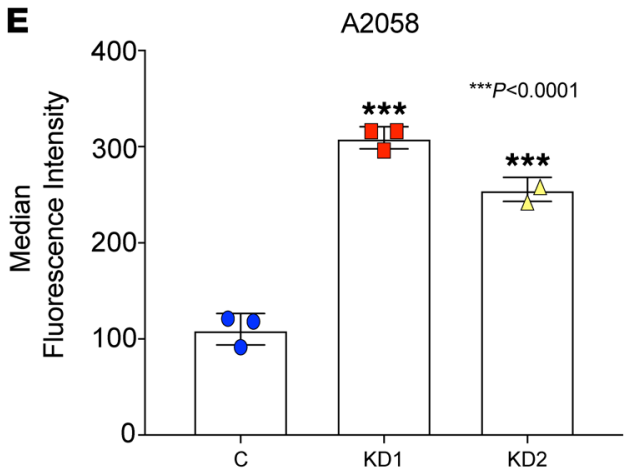

G

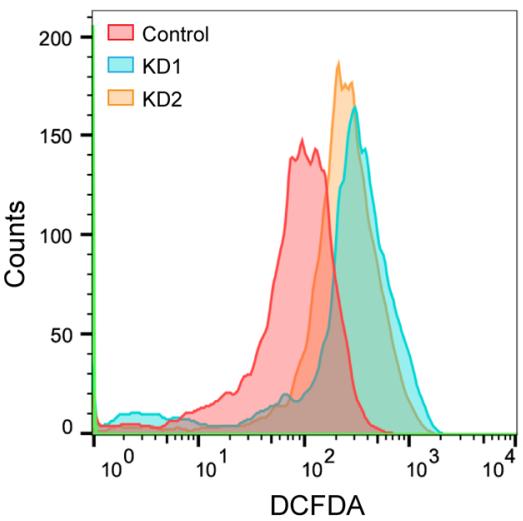

$\mathbf{F}$

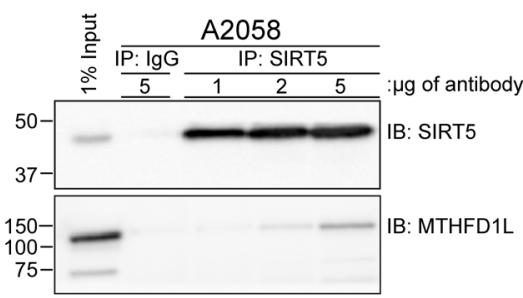


Figure 9. SIRT5 promotes histone methylation and reduced cellular ROS levels in melanoma. (A) LC-MS/MS-based metabolite profiling followed by MetaboAnalyst pathway analysis demonstrate alterations in glycine and serine and methionine biosynthesis pathways in melanoma cells upon SIRT5 depletion. (B) Perturbations in 1C metabolite levels in response to SIRT5 loss in the cell lines shown. Each column represents the mean of 3 independently prepared biological replicates. Metabolite levels in SIRT5-depleted (KD1 and KD2, as indicated) samples are normalized to control. SAM, S-adenosyl-methionine; SAH, S-adenosylhomocysteine; GSH, reduced glutathione; CSSG, glutathione disulfide. (C) H3K4me3 and H3K9me3 immunoblot in melanoma cells 96 hours after transduction with shRNAs SIRT5-KD1 or -KD2 compared with a nontargeting control. (D) H3K4me3 and H3K9me3 levels are restored in A2058 cells lacking SIRT5 after 4 weeks of continual culture in puromycin. (E) Flow cytometric analysis of DCFDA-stained A2058 cells 96 hours after transduction with shRNAs SIRT5KD1 or $-\mathrm{KD} 2$ reveals increased ROS compared with a nontargeting control, $P$ $<0.005$. Left panel, average mean fluorescence intensity of DCFDA positive populations in $n=3$ samples. Error bars represent standard deviation. Significance calculated using 1-way ANOVA. Right panel, representative ( $n$ = 6) flow cytometric analysis of A2058 cells stained with DCFDA. (F) SIRT5 interacts with MTHFD1L in A2058 cells. Increasing amounts of anti-SIRT5 antibody increases SIRT5-MTHFD1L coprecipitation compared with normal rabbit IgC control. Basal expression of SIRT5 and MTHFD1L in whole-cell extract ( $1 \%$ of initial amount used for immunoprecipitation) is shown for comparison. (C) Proposed model of promotion of MITF and c-MYC expression via SIRT5-dependent chromatin modifications in human melanoma. $\mathrm{Me}$, methylation; Ac, acetylation. C, control.

with these data, we observed a loss of MITF expression and a concomitant reduction in expression of $c-M Y C$ in SIRT5-depleted melanoma cell lines. A positive correlation between SIRT5 and $c$-MYC RNA expression in melanoma tumors from TCGA data was observed (Supplemental Figure 6C). Both c-MYC RNA and c-MYC protein levels were decreased in melanoma cells after SIRT5 ablation (Supplemental Figure 6, D and E).

Gene set enrichment analysis (GSEA) was used to identify pathways affected by SIRT5 depletion. GSEA revealed negatively enriched gene patterns in $c-M Y C$, c-MYC-target gene signatures, and mitochondrial biogenesis pathways (Supplemental Figure $6 \mathrm{~F}$ ). We also observed a positive enrichment of genes involved in apoptosis, consistent with our observation that SIRT5 loss induces apoptosis in melanoma cells (see Figure 3). Ingenuity Pathway Analysis (IPA) of transcriptional regulators predicts that both MITF and $c-M Y C$ were significantly inhibited by SIRT5 depletion, based on comparisons between data from aggregated SIRT5-KD melanoma cells and SIRT5 control lines (Supplemental Figure 6G). The multiple canonical pathways altered upon SIRT5 loss highlight other, pleiotropic effects of SIRT5 depletion on melanoma cells (Supplemental Figure 6H). Taken together, these data show that SIRT5 promotes expression and activity of 2 key oncogenic drivers, MITF and c-MYC, in melanoma.

SIRT5 regulates melanoma cell metabolism to promote histone acetylation. To obtain systems-level insight into potential roles for SIRT5 in regulating gene expression, we reanalyzed our transcriptomic data using a genome-scale model of human metabolism to identify metabolic reactions that change in activity after SIRT5 KD. The Recon1 human network model used contains a relationship between 3744 reactions, 2766 metabolites, 1496 metabolic genes, and 2004 metabolic enzymes (69). This network model has been used successfully to predict the metabolic behavior of various cancer cells and stem cells $(70,71)$. Using this model, we identified a metabolic flux state most consistent with expression data for each of the 3 cell lines after SIRT5 depletion. This was achieved by maximizing the activity of reactions that are associated with upregulated genes and minimizing flux through reactions that are downregulated for each condition, while simultaneously satisfying the stoichiometric and thermodynamic constraints embedded in the model (see Methods).

The model identified 20 reactions among the 3744 that showed significantly different activity across all cell lines after SIRT5 KD $(P<0.01$; Figure 8A and Supplemental Table 4). Among these, the enzyme ATP-citrate lyase (ACLY) was predicted to have the most significant change, with reduced activity after SIRT5 KD. ACLY generates acetyl-CoA from citrate, thereby playing an important role in supporting histone acetylation (72). Furthermore, the mitochondrial methylenetetrahydrofolate dehydrogenase reaction was also predicted to have reduced activity after SIRT5 loss, a part of the folate and one-carbon metabolism (1CM) pathways (see below). Several reactions involving cholesterol metabolism and nucleotide salvage were also affected by SIRT5 KD, highlighting the pervasive effects of SIRT5 in melanoma cells.

To test the predictions of the metabolic model, we evaluated protein acetylation levels in SIRT5 KD cells. Indeed, SIRT5 depletion induced a striking decrease in total lysine acetylation, most notably on histones, including H3K9 acetylation (H3K9ac) and H4K16 acetylation (H4K16ac; Figure 8, B and C). This reduction in H3K9ac, a known mark of active gene expression (73), combined with the decrease in MITF and c-MYC, prompted us to test whether H3K9ac levels are reduced within the promoter regions of these genes. CUT\&RUN (cleavage under targets and release using nuclease) followed by qRT-PCR in A2058 cells demonstrated that upon SIRT5 depletion, a significant reduction of $\mathrm{H} 3 \mathrm{~K} 9 \mathrm{ac}$ in the promoter regions of both MITF and $c-M Y C$ occurred (Figure $8 \mathrm{D}$ ), suggesting a role for SIRT5 in maintaining transcriptional activity of these genes in melanoma cells by promoting histone acetylation.

After 4 weeks in culture following SIRT5 KD, a small residual population of A2058 cells overcame SIRT5 loss-of-function to survive and proliferate, although SIRT5 depletion was maintained. Importantly, total lysine acetylation and MITF expression was restored in surviving SIRT5-KD A2058 cell populations (Figure 8, E and F), consistent with the relevance of SIRT5-driven histone acetylation in melanoma survival. This phenotype was recapitulated in vivo. Although tumors that formed in SIRT5-deficient BrafCA Pten ${ }^{\mathrm{Al}}$ ${ }^{f l}$ Tyr:CreER mice were smaller than WT controls (see Figure 4), total lysine acetylation, H3K9ac, MITF, and c-MYC protein levels were similar to controls. Markers for cell death (PARP cleavage) and cellular proliferation (PCNA and phospho-histone H3 S10 [H3pS10]) were also similar between SIRT5-WT and SIRT5-deficient tumors in the model, suggesting that these parameters may have recovered during successful tumor formation (Supplemental Figure 7D).

Protein acetyltransferases employ acetyl-CoA to acetylate their protein targets, including histones (74). To investigate the potential basis for reduced histone acetylation in SIRT5-depleted melanoma cells, we employed a sensitive mass spectrometry-based method to assess total cellular acetyl-CoA levels $(75,76)$. Surprisingly, we observed an increase of total cellular acetyl-CoA after SIRT5 KD (Figure 8G), implying that reduced acetyl-CoA levels do not con- 
tribute to the observed decrease in lysine acetylation upon SIRT5 depletion, and suggesting that other phenomena, such as reduced acetyltransferase activity, may underlie the reduced acetylation levels in SIRT5-depleted melanoma cells (see Discussion).

SIRT5 promotes 1CM and histone methylation in melanoma. To investigate further how SIRT5 may function to affect gene expression in melanoma, SIRT5-depleted melanoma cell lines were profiled using liquid chromatography coupled tandem mass spectrometry-based (LC-MS/MS-based) metabolomics, followed by functional analysis using MetaboAnalyst pathway enrichment (Supplemental Table 5). Two BRAF mutant lines (A2058 and A375) and an NRAS mutant (SK-MEL-2) showed perturbations in pathways involving $1 \mathrm{CM}$ in response to SIRT5 depletion (Figure 9A and Supplemental Figure 7, A and B). 1CM is comprised of the linked folate and methionine cycles (77). Outputs include metabolites required for amino acid and nucleotide synthesis, glutathione for antioxidant defense, and crucially, S-adenosylmethionine (SAM) for methylation reactions, including those on histones. We observed a reduction in levels of several key 1CM metabolites upon SIRT5 depletion in BRAF mutant melanoma cell lines, but not in SK-MEL-2 (Figure 9B).

Histone methylation, particularly $\mathrm{H} 3 \mathrm{~K} 4$ trimethylation (H3K4me3), is highly sensitive to fluctuations in SAM levels (78). We observed reductions in $\mathrm{H} 3 \mathrm{~K} 4 \mathrm{me} 3$ and $\mathrm{H} 3 \mathrm{~K} 9 \mathrm{me} 3$ in melanoma cells following SIRT5 KD, consistent with 1CM perturbation (Figure 9C). However, addition of exogenous SAM did not consistently restore $\mathrm{H} 3 \mathrm{~K} 4 \mathrm{me} 3$ or $\mathrm{H} 3 \mathrm{~K} 9 \mathrm{me} 3$, nor did it markedly elevate levels of these marks in control cells (Supplemental Figure 7C and not shown). As for acetylation, SIRT5-depleted melanoma cells that grew out after prolonged culture recovered H3K4me3 and H3K9me3 levels (Figure 9D), while maintaining reduced SIRT5 expression (Figure 8E), suggesting that loss of these histone modifications represents an important driver of the lethality associated with SIRT5 depletion in melanoma.

A decrease in cellular glutathione content occurring in the context of impaired 1CM would be predicted to elevate levels of cellular ROS (79). Consistently, in A2058 cells, we observed increased staining with $2^{\prime}, 7^{\prime}$-dichlorofluorescin diacetate (DCFDA), a ROS-sensitive dye, following SIRT5 depletion (Figure 9E). However, treatment with the antioxidants, N-acetylcysteine, mitoTEMPOL, or $\beta$-mercaptoethanol failed to mitigate cell lethality after SIRT5 loss (unpublished observation), indicating that regulation of ROS levels is not likely a primary determinant of the requirement of melanoma cells for SIRT5.

We noted that previous proteomic surveys identified the $1 \mathrm{CM}$ enzyme, MTHFD1L (methylenetetrahydrofolate dehydrogenase [NADP ${ }^{+}$dependent 1-like]), as a candidate SIRT5 substrate (11, 80). MTHFD1L is a $1 \mathrm{CM}$ enzyme that participates in the folate cycle to convert formate and tetrahydrofolate into 10-formyl-tetrahydrofolate in an ATP-dependent reaction. We tested the interaction of MTHFD1L with SIRT5 in the context of melanoma, and found that MTHFD1L coimmunoprecipitates with SIRT5 (Figure 9F). These data suggest a potential role for SIRT5 in regulating multiple 1CM enzymes, such as SHMT2 and potentially MTHFD1L and others, to promote $1 \mathrm{CM}$ and histone methylation. Likewise, since SK-MEL-2 cells showed a reduction in histone H3K4me3 levels without apparent declines in 1C metabolites under our experimental conditions, it is likely that SIRT5 plays additional roles in regulating histone methylation, perhaps in an oncogenic driver-dependent manner. We propose that SIRT5 regulates histone methylation and acetylation via regulation of multiple protein targets in melanoma cells.

\section{Discussion}

Sirtuin-family $\mathrm{NAD}^{+}$-dependent protein deacylases regulate metabolism and other diverse aspects of cell biology (81). SIRT5 is a poorly understood, atypical sirtuin, whose primary known biochemical function is to remove succinyl, malonyl, and glutaryl groups from lysines on its target proteins (8, 9, 11-13). A substantial fraction of SIRT5 is present in the mitochondrial matrix; however, SIRT5 is present and functional in the cytosol, and even in the nucleus $(11,14)$. Most of the phenotypes associated with SIRT5 loss-of-function in normal cells and tissues reported in the literature to date are remarkably mild (17). In sharp contrast, here we report that cutaneous and uveal melanoma cells show exquisite dependency on SIRT5, in a genotype-independent manner. SIRT5 depletion, induced by shRNA or CRISPR/Cas9, provokes dramatic, rapid loss of cell viability and induction of apoptosis in both cutaneous and uveal melanoma cell lines. Likewise, SIRT5 promotes melanoma xenograft tumor formation in immunocompromised mice, and melanoma formation in an autochthonous Braf Pten-driven mouse melanoma strain.

Our transcriptomic analyses reveal that SIRT5 plays a major role in maintaining proper gene expression in melanoma cells. SIRT5-dependent genes notably include the lineage-specific oncogenic transcription factor MITF (82) and $c-M Y C$ (41). In the TCGA data set, SIRT5 levels correlate with those of MITF and $c-M Y C$, suggesting that SIRT5 activity influences both MITF and $c-M Y C$ expression in a physiologic context. Indeed, we found that SIRT5 depletion results in loss of H3K9ac, a marker for active transcription, within the promoter regions of these genes. These data are consistent with previously published results describing a role for histone modifications in sustaining MITF expression and melanoma proliferation (83). Genetic or pharmaceutical inhibition of the p300 acetyltransferase results in reduced MITF expression, reduced histone acetylation within of the MITF promoter, and induction of markers of cellular senescence in melanoma cell lines, suggesting regulation of chromatin dynamics as a mechanism of MITF expression and melanoma growth (83). Via metabolomic analysis, we identified a role for SIRT5 in promoting $1 \mathrm{CM}$ in 2 BRAF-dependent cell lines, and in maintaining histone trimethylation at $\mathrm{H} 3 \mathrm{~K} 4$ and $\mathrm{H} 3 \mathrm{~K}$ 9, marks associated with transcriptional activation and repression, respectively. SIRT5 also plays a distinct role in maintaining histone acetylation. To our knowledge, SIRT5 is the first protein implicated in maintaining both histone methylation and acetylation, highlighting its important roles in maintaining chromatin structure and gene expression in melanoma.

Our in vivo findings in an autochthonous system are in contrast to a published study by Moon et al., in which SIRT5 deficiency was found to exert no impact on tumor growth in a similar mouse melanoma model as the one used in our studies (84). Several potential explanations exist for this discrepancy. Moon et al. used a Sirt 5 allele distinct from the one employed in our work. The Sirt5 allele used in their analysis deletes a single exon in the Sirt5 gene 
(16), whereas the one used herein deletes essentially the entire Sirt5 protein coding sequence (15). Likewise, subtle genetic background differences in the strains of the mice used may contribute to these discrepancies, as could microbiome differences between the mouse colonies. Another potential explanation involves the protocol used to induce gene recombination; we applied a higher concentration of tamoxifen than did Moon et al. (64.5 mM vs. 5 $\mathrm{mM}$ ). Importantly, since our model is a global Sirt5-KO, we cannot rule out the possibility that SIRT5 may function melanoma-cell nonautonomously in this system, for example, by modulating the antimelanoma immune response or other aspects of the tumor microenvironment. However, given the striking dependency of cultured melanoma cells on SIRT5 in vitro, we strongly suspect that a very important component of SIRT5's function, at minimum, is a cell-autonomous prosurvival role in melanoma cells.

MITF is a member of the microphthalmia family of transcription factors, and is dysregulated in melanoma (85). Attenuation of melanocyte differentiation and pigmentation are observed in humans and mice deficient for MITF activity, highlighting the importance of MITF in melanocyte survival and function. Likewise, MITF is known to play key roles in melanoma cell survival and differentiation, and MITF amplification occurs in 15\% to $20 \%$ of melanomas, associated with a worsened prognosis (64). In melanoma cell lines where MITF is expressed, SIRT5 depletion induced a rapid decrease in expression of MITF itself and several well-characterized MITF targets. Likewise, in TCGA data, SIRT5 and MITF levels were highly correlated, suggesting that SIRT5 may play a role in regulating MITF in tumors in vivo. Notably, we were unable to rescue the lethality of SIRT5 depletion by overexpressing MITF in melanoma cells (unpublished observation). However, this experiment is complicated by the fact that MITF overexpression itself can drive melanoma cells to leave the cell cycle and differentiate, and thus is likely selected against in shortterm culture (86). Likewise, we were unable to rescue SIRT5-depleted melanoma cells via c-MYC overexpression, although we were able to overexpress c-MYC (unpublished observation). Nevertheless, given the well-known importance of these transcription factors in melanoma pathobiology, we hypothesize that loss of MITF and c-MYC expression likely represent important mechanisms through which SIRT5 promotes melanoma viability.

We did not observe major effects of SIRT5 depletion on OCR, ECAR, or overall ATP production in melanoma. Instead, through mass spectrometry-based metabolite profiling, we identified 1CM as one SIRT5 target pathway likely important for maintenance of gene expression and melanoma viability. 1CM consists of the linked folate and methionine cycles. A major output of $1 \mathrm{CM}$ is SAM, the universal methyl donor in mammalian cells. Metabolite profiling in 2 BRAF mutant melanoma cells lacking SIRT5 reveals profound perturbations in levels of many $1 \mathrm{C}$ metabolites, including reductions in cellular SAM. Moreover, H3K4me3, a mark of active gene expression and a sensitive marker for intracellular SAM levels, drops in response to SIRT5 loss-of-function. Furthermore, global lysine acetylation and $\mathrm{H} 3 \mathrm{~K} 9 \mathrm{me} 3$, which marks heterochromatic regions in the genome (87) decrease upon SIRT5 loss. Likewise, oxidative stress increases in SIRT5-depleted melanoma cells, consistent with impaired regeneration of reduced glutathione, a major antioxidant species and an output of $1 \mathrm{CM}$.
Many open questions remain as to the mechanisms by which SIRT5 promotes proper gene expression and viability in melanoma. The accumulation of acetyl-CoA in SIRT5-depleted melanoma cells suggests that SIRT5 may promote the activity of a histone acetyltransferase to promote histone acetylation, a possibility that we are currently investigating. Alternatively, SIRT5 could promote generation of a localized acetyl-CoA pool necessary to drive histone acetylation (compare to the nuclear pool, ref. 74), without influencing global acetyl-CoA levels. A large number of studies implicate alterations in levels of specific metabolites in driving chromatin modifications (88). Increased lactate, for example, inhibits histone deacetylases, thereby increasing histone acetylation (89). Although we observe only modest and, in some cases, inconsistent changes in cellular metabolite levels upon SIRT5 KD, it is possible that alterations in levels of specific metabolites, or a combination of these metabolite abnormalities, may in part be responsible for the loss of histone modifications we observe. In addition, we identified MTHFD1L as a SIRT5 interactor and candidate target that may play a role in SIRT5-mediated regulation of $1 \mathrm{CM}$. Unfortunately, we have been unsuccessful at rescuing the cellular lethality associated with SIRT5 depletion using relevant small molecule metabolites or drugs (acetate, acetyl-CoA, SAM, serine, glycine, histone deacetylase and demethylase inhibitors, antioxidants, nucleotides, and amino acids [unpublished observations]). We suspect that this reflects pleiotropic functions and targets of SIRT5 in melanoma cells, impairment of which cannot be rescued by intervention in any individual pathway. SIRT5 targets involved in other pathways - e.g., ROS suppression, cell death $(32,90)$, and others - could well contribute to the requirement of melanoma cells for SIRT5. Likewise, we identified perturbations in innate immune pathways in SIRT5-depleted melanoma cells, which could also contribute to the requirement of melanoma cells for this protein. This is consistent with the hundreds of cellular targets of SIRT5, involved in diverse cellular pathways, identified in proteomics studies (17). Moreover, it is consistent with the observation that SIRT5 plays prosurvival roles across multiple different cancer types, via distinct proposed mechanisms. As the dominant cellular desuccinylase/demalonylase/deglutarylase, it is possible that SIRT5 is recruited to play distinct roles in supporting tumorigenesis, modulating activities of different suites of targets and pathways, in a cancer type-specific manner.

Overall, our data reveal a major, hitherto unknown requirement for SIRT5 in melanoma cell survival, through suppression of apoptosis via regulation of chromatin modifications and expression of critical prosurvival genes, including MITF and c-MYC (Figure $9 \mathrm{G}$ ). These results, along with those already in the literature (7), suggest that SIRT5 may play potent oncogenic roles across many diverse tumor types, seemingly engaging a variety of different cellular mechanisms to do so in a cancer- and context-specific manner. Since the phenotypes of Sirt 5 null mice are quite mild, we propose that SIRT5 may represent an attractive therapeutic target, in melanoma and specific other cancer types. In this regard, published studies (17, 91-94), including recent work focused on breast cancer (37), demonstrate that SIRT5 is in principle druggable with small molecules. SIRT5 dependency may be particularly translationally significant in uveal melanoma, where currently no effective therapeutic options exist for patients with metastatic disease. 


\section{Methods}

Transcriptomic analysis of SIRT5 depletion. RNA-Seq data are publicly available (GEO accession number GSE169205) for download from the GEO website (http://www.ncbi.nlm.nih.gov/geo/).

Study approval. All mice were housed at the Biomedical Science Research Building, University of Michigan (UM). All vertebrate animal experiments were approved by and performed in accordance with the regulations of the University Committee on Use and Care of Animals.

\section{Author contributions}

WG, LBR, AHG, SK, ACM, AMM, MES, MA, ASAM, CHC, NK, KAM, HJL, LZ, PS, ST, ELV, SI, MW, JSW, HPS, RA Sturm, ALP, AAA, RA Scolyer, MSS, DAS, DRF, MWB, SC, ZNC, MEV, NWS, MHR, ALO, and CAL performed experiments and/or analyzed data. WG and DBL interpreted data, and wrote and revised the paper. WG made the figures. DBL supervised the overall design and study interpretation.

\section{Acknowledgments}

This work was supported in part by the Melanoma Research Alliance (to DL and CAL), NIH R01GM101171, AACR-Bayer (17-8044-LOMB), DoD awards CA170628, CA190267, OC140123, and NF170044 (to DL), and the Rogel Cancer Center. Use of the Rogel Cancer Center shared resources is also gratefully acknowledged by DL and CAL (P30CA46592). SK was supported in part through an award from the Pablove Foundation. CAL was supported by a 2017 AACR NextGen Grant for Transformative Cancer Research (17-20-01-LYSS), an ACS Research Scholar Grant (RSG-18-18601), and NIH award R01CA244931. ZNC was supported by NIH R01CA217141 and AACR-Bayer (19-80-44-NIKO). Metabolom- ics studies performed at the University of Michigan were supported by NIH grant DK097153. ALP is supported by the Highlands and Islands Enterprise (HMS9353763). MSS holds a fellowship (APP1106491) from the National Health and Medical Research Council (NHMRC). WG, AG, AM, LR, and PS were supported by NIH T32 awards (WG: GM007544, AG000114, HL007853 and AR007917; AG: AG000114 and GM113900; AM: AG000114; LR: NL007517; PS: CA009676). NWS was supported by R01GM132261. ST was funded by American Diabetes Association postdoctoral fellowship 1-18-PDF-144. RAS and JSW are supported by the Australian National Health and Medical Research Council Fellowship program. RAS is also supported by a NHMRC of Australia Program Grant (APP1093017). Support from colleagues at Melanoma Institute Australia and the Royal Prince Alfred Hospital is also gratefully acknowledged. HPS holds an NHMRC MRFF Next Generation Clinical Researchers Program Practitioner Fellowship (APP1137127). The SBP Cancer Metabolism Core is supported by National Cancer Institute Cancer Center Support Grant P30 030199. We acknowledge Jeongsoon Park (UM) for technical contributions early in the project; Scott Pletcher (UM) for assistance with statistical analysis; Emily Bernstein (Icahn School of Medicine at Mount Sinai) and Kathleen Cho (UM) for generously providing cell lines; David Fisher (MGH Cancer Center/Harvard Medical School) and Kathryn Wellen (University of Pennsylvania) for helpful discussions; Olga Zagnitko for assistance with GC/MS measurements; and Robert Weiss, Hening Lin, and Michael Deininger for discussion of unpublished data.

Address correspondence to: David B. Lombard, University of Michigan, 3015 BSRB, 109 Zina Pitcher Place, Ann Arbor, Michigan 48109, USA. Phone: 734.615.0498; Email: davidlom@med.umich.edu.
1. Siegel RL, et al. Cancer Statistics, 2021. CA Cancer J Clin. 2021;71(1):7-33.

2. Siegel RL, et al. Cancer statistics, 2019. CA Cancer J Clin. 2019;69(1):7-34.

3. Dharmadhikari N, et al. Oncolytic virus immunotherapy for melanoma. Curr Treat Options Oncol. 2015;16(3):326.

4. Guy GP, Ekwueme DU. Years of potential life lost and indirect costs of melanoma and non-melanoma skin cancer: a systematic review of the literature. Pharmacoeconomics. 2011;29(10):863-874.

5. Larkin J, et al. Five-year survival with combined nivolumab and ipilimumab in advanced melanoma. N Engl JMed. 2019;381(16):1535-1546.

6. Singh M, et al. Uveal melanoma: a review of the literature. Oncol Ther. 2018;6(1):87-104.

7. Bringman-Rodenbarger LR, et al. Emerging roles for SIRT5 in metabolism and cancer. Antioxid Redox Signal. 2018;28(8):677-690.

8. Du J, et al. Sirt5 is a NAD-dependent protein lysine demalonylase and desuccinylase. Science. 2011;334(6057):806-809.

9. Rardin MJ, et al. SIRT5 regulates the mitochondrial lysine succinylome and metabolic networks. Cell Metab. 2013;18(6):920-933.

10. North BJ, et al. The human Sir2 ortholog, SIRT2, is an NAD+-dependent tubulin deacetylase. $\mathrm{Mol}$ Cell. 2003;11(2):437-444.

11. Park J, et al. SIRT5-mediated lysine desuccinylation impacts diverse metabolic pathways. $\mathrm{Mol}$
Cell. 2013;50(6):919-930.

12. Tan M, et al. Lysine glutarylation is a protein posttranslational modification regulated by SIRT5. Cell Metab. 2014;19(4):605-617.

13. Peng $\mathrm{C}$, et al. The first identification of lysine malonylation substrates and its regulatory enzyme. $\mathrm{Mol}$ Cell Proteomics. 2011;10(12):M111 012658 1-12.

14. Nishida Y, et al. SIRT5 regulates both cytosolic and mitochondrial protein malonylation with glycolysis as a major target. Mol Cell. 2015;59(2):321-332.

15. Lombard DB, et al. Mammalian Sir2 homolog SIRT3 regulates global mitochondrial lysine acetylation. Mol Cell Biol. 2007;27(24):8807-8814.

16. Yu J, et al. Metabolic characterization of a Sirt5 deficient mouse model. Sci Rep. 2013;3:2806.

17. Kumar S, Lombard DB. Functions of the sirtuin deacylase SIRT5 in normal physiology and pathobiology. Crit Rev Biochem Mol Biol. 2018;53(3):311-334

18. Boylston JA, et al. Characterization of the cardiac succinylome and its role in ischemia-reperfusion injury. J Mol Cell Cardiol. 2015;88:73-81.

19. Sadhukhan S, et al. Metabolomics-assisted proteomics identifies succinylation and SIRT5 as important regulators of cardiac function. Proc Natl Acad Sci U S A . 2016;113(16):4320-4325.

20. Hershberger KA, et al. Sirtuin 5 is required for mouse survival in response to cardiac pressure overload. J Biol Chem. 2017;292(48):19767-19781.
21. Hershberger KA, et al. Ablation of Sirtuin 5 in the postnatal mouse heart results in protein succinylation and normal survival in response to chronic pressure overload. J Biol Chem. 2018;293(27):10630-10645.

22. Roth M, Chen WY. Sorting out functions of sirtuins in cancer. Oncogene. 2014;33(13):1609-1620

23. Ohanna M, et al. SIRT1 promotes proliferation and inhibits the senescence-like phenotype in human melanoma cells. Oncotarget. 2014;5(8):2085-2095.

24. Bajpe PK, et al. A chromatin modifier genetic screen identifies SIRT2 as a modulator of response to targeted therapies through the regulation of MEK kinase activity. Oncogene. 2015;34(4):531-536.

25. George J, et al. Pro-proliferative function of mitochondrial sirtuin deacetylase SIRT3 in human melanoma. J Invest Dermatol. 2016;136(4):809-818.

26. Garcia-Peterson LM, et al. SIRT6 histone deacetylase functions as a potential oncogene in human melanoma. Genes Cancer. 2017;8(9-10):701-712.

27. Strub T, et al. SIRT6 haploinsufficiency induces BRAF(V600E) melanoma cell resistance to MAPK inhibitors via IGF signalling. Nat Commun. 2018;9(1):3440.

28. Lu W, et al. SIRT5 facilitates cancer cell growth and drug resistance in non-small cell lung cancer. Tumour Biol. 2014;35(11):10699-10705.

29. Lv XB, et al. SUN2 exerts tumor suppressor func- 
tions by suppressing the Warburg effect in lung cancer. Sci Rep. 2015;5:17940.

30. Lin ZF, et al. SIRT5 desuccinylates and activates SOD1 to eliminate ROS. Biochem Biophys Res Commun. 2013;441(1):191-195.

31. Zhou L, et al. SIRT5 promotes IDH2 desuccinylation and G6PD deglutarylation to enhance cellular antioxidant defense. EMBO Rep. 2016;17(6):811-822.

32. Yang X, et al. SHMT2 desuccinylation by SIRT5 drives cancer cell proliferation. Cancer Res. 2018;78(2):372-386.

33. Chang L, et al. SIRT5 promotes cell proliferation and invasion in hepatocellular carcinoma by targeting E2F1. Mol Med Rep. 2018;17(1):342-349.

34. Zhang R, et al. SIRT5 promotes hepatocellular carcinoma progression by regulating mitochondrial apoptosis. J Cancer. 2019;10(16):3871-3882 .

35. Greene KS, et al. SIRT5 stabilizes mitochondrial glutaminase and supports breast cancer tumorigenesis. Proc Natl Acad Sci U S A. 2019;116(52):26625-26632.

36. Pavlova NN, Thompson CB. The emerging hallmarks of cancer metabolism. Cell Metab. 2016;23(1):27-47.

37. Abril YLN, et al. Pharmacological and genetic perturbation establish SIRT5 as a promising target in breast cancer. Oncogene. 2021;40(9):1644-1658.

38. Li F, et al. NADP(+)-IDH mutations promote hypersuccinylation that impairs mitochondria respiration and induces apoptosis resistance. $\mathrm{Mol}$ Cell. 2015;60(4):661-675.

39. Xu YS, et al. STAT3 Undergoes acetylationdependent mitochondrial translocation to regulate pyruvate metabolism. Sci Rep. 2016;6:39517.

40. Hartman ML, Czyz M. Pro-survival role of MITF in melanoma. JInvest Dermatol. 2015;135(2):352-358.

41. Zhuang D, et al. C-MYC overexpression is required for continuous suppression of oncogene-induced senescence in melanoma cells. Oncogene. 2008;27(52):6623-6634.

42. Singleton KR, et al. Melanoma therapeutic strategies that select against resistance by exploiting MYC-driven evolutionary convergence. Cell Rep. 2017;21(10):2796-2812.

43. North JP, et al. Assessment of copy number status of chromosomes 6 and 11 by FISH provides independent prognostic information in primary melanoma. Am J Surg Pathol. 2011;35(8):1146-1150.

44. Santos GC, et al. Chromosome 6p amplification and cancer progression. J Clin Pathol. 2007;60(1):1-7.

45. Cancer Genome Atlas Network. Genomic classification of cutaneous melanoma. Cell. 2015;161(7):1681-1696.

46. Hodis $\mathrm{E}$, et al. A landscape of driver mutations in melanoma. Cell. 2012;150(2):251-263.

47. Wu D, et al. Advances in cellular characterization of the sirtuin isoform, SIRT7. Front Endocrinol (Lausanne). 2018;9:652.

48. Stark M, Hayward N. Genome-wide loss of heterozygosity and copy number analysis in melanoma using high-density single-nucleotide polymorphism arrays. Cancer Res. 2007;67(6):2632-2642.

49. Dutton-Regester K, et al. Identification of TFG (TRK-fused gene) as a putative metastatic melanoma tumor suppressor gene. Genes Chromo- somes Cancer. 2012;51(5):452-461.

50. Stark MS, et al. Whole-exome sequencing of acquired nevi identifies mechanisms for development and maintenance of benign neoplasms. J Invest Dermatol. 2018;138(7):1636-1644.

51. Shain AH, et al. The genetic evolution of melanoma from precursor lesions. $N$ Engl J Med. 2015;373(20):1926-1936.

52. Luebker SA, Koepsell SA. Diverse mechanisms of BRAF inhibitor resistance in melanoma identified in clinical and preclinical studies. Front Oncol. 2019;9:268.

53. Meeth K, et al. The YUMM lines: a series of congenic mouse melanoma cell lines with defined genetic alterations. Pigment Cell Melanoma Res. 2016;29(5):590-597.

54. Hartman ML. Non-apoptotic cell death signaling pathways in melanoma. Int J Mol Sci. 2020;21(8):E2980.

55. Dankort D, et al. Braf(V600E) cooperates with Pten loss to induce metastatic melanoma. Nat Genet. 2009;41(5):544-552.

56. Buler M, et al. SIRT5 is under the control of PGC- $1 \alpha$ and AMPK and is involved in regulation of mitochondrial energy metabolism. FASEB J. 2014;28(7):3225-3237.

57. Zhang Y, et al. Lysine desuccinylase SIRT5 binds to cardiolipin and regulates the electron transport chain. J Biol Chem. 2017;292(24):10239-10249.

58. Scott DA, et al. Comparative metabolic flux profiling of melanoma cell lines: beyond the Warburg effect. JBiol Chem. 2011;286(49):42626-42634.

59. Filipp FV, et al. Reverse TCA cycle flux through isocitrate dehydrogenases 1 and 2 is required for lipogenesis in hypoxic melanoma cells. Pigment Cell Melanoma Res. 2012;25(3):375-383.

60. Filipp FV, et al. Glutamine-fueled mitochondrial metabolism is decoupled from glycolysis in melanoma. Pigment Cell Melanoma Res. 2012;25(6):732-739.

61. Ratnikov B, et al. Glutamate and asparagine cataplerosis underlie glutamine addiction in melanoma. Oncotarget. 2015;6(10):7379-7389.

62. Qin JZ, et al. Targeting glutamine metabolism sensitizes melanoma cells to TRAIL-induced death. Biochem Biophys Res Commun. 2010;398(1):146-152.

63. Qin JZ, et al. 2-deoxyglucose sensitizes melanoma cells to TRAIL-induced apoptosis which is reduced by mannose. Biochem Biophys Res Commun. 2010;401(2):293-299.

64. Garraway LA, et al. Integrative genomic analyses identify MITF as a lineage survival oncogene amplified in malignant melanoma. Nature. 2005;436(7047):117-122.

65. Hoek KS, Goding CR. Cancer stem cells versus phenotype-switching in melanoma. Pigment Cell Melanoma Res. 2010;23(6):746-759.

66. Müller J, et al. Low MITF/AXL ratio predicts early resistance to multiple targeted drugs in melanoma. Nat Commun. 2014;5(1):5712.

67. Cheli Y, et al. Mitf is the key molecular switch between mouse or human melanoma initiating cells and their differentiated progeny. Oncogene. 2011;30(20):2307-2318

68. Seoane M, et al. Lineage-specific control of TFIIH by MITF determines transcriptional homeostasis and DNA repair. Oncogene.
2019;38(19):3616-3635.

69. Duarte NC, et al. Global reconstruction of the human metabolic network based on genomic and bibliomic data. Proc Natl Acad Sci U S A. 2007;104(6):1777-1782.

70. Chandrasekaran S, et al. Comprehensive mapping of pluripotent stem cell metabolism using dynamic genome-scale network modeling. Cell Rep. 2017;21(10):2965-2977.

71. Shlomi T, et al. Network-based prediction of human tissue-specific metabolism. Nat Biotechnol. 2008;26(9):1003-1010.

72. Wellen KE, et al. ATP-citrate lyase links cellular metabolism to histone acetylation. Science. 2009;324(5930):1076-1080.

73. Gates LA, et al. Acetylation on histone H3 lysine 9 mediates a switch from transcription initiation to elongation. J Biol Chem. 2017;292(35):14456-14472.

74. Sivanand S, et al. Spatiotemporal control of acetyl-CoA metabolism in chromatin regulation. Trends Biochem Sci. 2018;43(1):61-74.

75. Snyder NW, et al. Production of stable isotopelabeled acyl-coenzyme A thioesters by yeast stable isotope labeling by essential nutrients in cell culture. Anal Biochem. 2015;474:59-65.

76. Frey AJ, et al. LC-quadrupole/orbitrap high-resolution mass spectrometry enables stable isotoperesolved simultaneous quantification and ${ }^{13} \mathrm{C}$-isotopic labeling of acyl-coenzyme A thioesters. Anal Bioanal Chem. 2016;408(13):3651-3658.

77. Yang M, Vousden KH. Serine and one-carbon metabolism in cancer. Nat Rev Cancer. 2016;16(10):650-662.

78. Gao X, et al. Metabolic interactions with cancer epigenetics. Mol Aspects Med. 2017;54:50-57.

79. Mailloux RJ, et al. Unearthing the secrets of mitochondrial ROS and glutathione in bioenergetics. Trends Biochem Sci. 2013;38(12):592-602.

80. Colak G, et al. Proteomic and biochemical studies of lysine malonylation suggest its malonic aciduria-associated regulatory role in mitochondrial function and fatty acid oxidation. Mol Cell Proteomics. 2015;14(11):3056-3071.

81. Kumar S, Lombard DB. Mitochondrial sirtuins and their relationships with metabolic disease and cancer. Antioxid Redox Signal. 2015;22(12):1060-1077.

82. Hartman ML, Czyz M. MITF in melanoma: mechanisms behind its expression and activity. Cell Mol Life Sci. 2015;72(7):1249-1260.

83. Kim E, et al. MITF expression predicts therapeutic vulnerability to $\mathrm{p} 300$ inhibition in human melanoma. Cancer Res. 2019;79(10):2649-2661.

84. Moon H, et al. Sirt5 is dispensable for Braf(V600E)-mediated cutaneous melanoma development and growth in vivo. Exp Dermatol. 2019;28(1):83-85.

85 . Haq R, Fisher DE. Biology and clinical relevance of the micropthalmia family of transcription factors in human cancer. JClin Oncol. 2011;29(25):3474-3482.

86. Wellbrock C, Marais R. Elevated expression of MITF counteracts B-RAF-stimulated melanocyte and melanoma cell proliferation. JCell Biol. 2005;170(5):703-708.

87. Becker JS, et al. H3K9me3-dependent heterochromatin: barrier to cell fate changes. Trends Genet. 2016;32(1):29-41. 
88. Li X, et al. Regulation of chromatin and gene expression by metabolic enzymes and metabolites. Nat Rev Mol Cell Biol. 2018;19(9):563-578.

89. Latham T, et al. Lactate, a product of glycolytic metabolism, inhibits histone deacetylase activity and promotes changes in gene expression. Nucleic Acids Res. 2012;40(11):4794-4803.

90. Schlicker C, et al. Substrates and regulation mechanisms for the human mitochon- drial sirtuins Sirt3 and Sirt5. J Mol Biol. 2008;382(3):790-801.

91. Roessler C, et al. Chemical probing of the human sirtuin 5 active site reveals its substrate acyl specificity and peptide-based inhibitors. Angew Chem Int Ed Engl. 2014;53(40):10728-10732.

92. Polletta L, et al. SIRT5 regulation of ammoniainduced autophagy and mitophagy. Autophagy. 2015;11(2):253-270.
93. Guetschow ED, et al. Identification of sirtuin 5 inhibitors by ultrafast microchip electrophoresis using nanoliter volume samples. Anal Bioanal Chem. 2016;408(3):721-731.

94. Yang LL, et al. Design and synthesis of new 9 -substituted norharmane derivatives as potential Sirt5 inhibitors. J Heterocycl Chem. 2017;54(2):1457-1466. 